\title{
Non-Gaussian Structure of B-mode Polarization after Delensing
}

\author{
Toshiya Namikawa ${ }^{a, b}$ and Ryo Nagata ${ }^{c}$ \\ ${ }^{a}$ Department of Physics, Stanford University, Stanford, CA 94305, USA \\ ${ }^{b}$ Kavli Institute for Particle Astrophysics and Cosmology, SLAC National Accelerator Lab- \\ oratory, Menlo Park, CA 94025, USA \\ ${ }^{c}$ High Energy Accelerator Research Organization (KEK), Tsukuba, Ibaraki 305-0801, Japan \\ E-mail: namikawa@slac.stanford.edu,rnagata@post.kek.jp
}

\begin{abstract}
The B-mode polarization of the cosmic microwave background on large scales has been considered as a probe of gravitational waves from the cosmic inflation. Ongoing and future experiments will, however, suffer from contamination due to the B-modes of nonprimordial origins, one of which is the lensing induced B-mode polarization. Subtraction of the lensing B-modes, usually referred to as delensing, will be required for further improvement of detection sensitivity of the gravitational waves. In such experiments, knowledge of statistical properties of the B-modes after delensing is indispensable to likelihood analysis particularly because the lensing B-modes are known to be non-Gaussian. In this paper, we study non-Gaussian structure of the delensed B-modes on large scales, comparing it with that of the lensing B-modes. In particular, we investigate the power spectrum correlation matrix and the probability distribution function (PDF) of the power spectrum amplitude. Assuming an experiment in which the quadratic delensing is an almost optimal method, we find that delensing reduces correlations of the lensing B-mode power spectra between different multipoles, and that the PDF of the power spectrum amplitude is well described as a normal distribution function with a variance larger than that in the case of a Gaussian field. These features are well captured by an analytic model based on the 4th order Edgeworth expansion. As a consequence of the non-Gaussianity, the constraint on the tensor-to-scalar ratio after delensing is degraded within approximately a few percent, which depends on the multipole range included in the analysis.
\end{abstract}




\section{Contents}

1 Introduction 1

2 Simulation Method 3

2.1 Map simulation 3

2.2 Quadratic lensing reconstruction 4

2.3 Quadratic delensing 5

3 Non-Gaussian Signature of Delensed B-mode Polarization 6

$\begin{array}{lll}3.1 & \text { Power spectrum covariance } & 6\end{array}$

$\begin{array}{lll}3.2 & \text { Probability distribution function of power spectrum amplitude } & 7\end{array}$

4 Modeling Statistical Properties of Delensed B-mode 8

4.1 Probability distribution function for weakly non-Gaussian fields 8

$\begin{array}{llr}4.2 & \text { Expression for B-mode power spectrum covariance } & 10\end{array}$

$\begin{array}{lll}4.3 & \text { Comparison with simulation results } & 11\end{array}$

5 Impact on Estimation of Tensor-to-Scalar Ratio 12

6 Summary and Discussion $\quad 14$

A Statistical Properties of Power Spectrum Amplitude $\mathbf{1 5}$

$\begin{array}{lll}\text { A.1 Mean } & 15\end{array}$

A.2 Variance 15

$\begin{array}{lll}\text { A.3 Probability distribution function of power spectrum amplitude } & 17\end{array}$

A.4 Formula of integration on unit hypersurface 20

B Delensed B-mode Power Spectrum Covariance 20

B.1 Analytic expression 20

B.2 Numerical computation 23

\section{Introduction}

Measurement of the B-mode polarization of the cosmic microwave background (CMB) on angular scales larger than a few dozen arcminutes has been considered as the best avenue to probe the primordial gravitational waves. The joint analysis of BICEP, Keck and PLANCK reported the constraint on the tensor-to-scalar ratio as $r_{0.05}<0.12$ (95\% C.L.) [1], while the much tighter constraint was recently obtained from PLANCK 2015 as $r_{0.002}<0.08(95 \%$ C.L.) [2], where the subscripts 0.05 and 0.002 are the pivot scale of the primordial power spectrum in unit of $\mathrm{Mpc}^{-1}$. Still, there is no evidence for the presence of the primordial gravitational waves.

On large scales, Galactic foreground emission, which is one of the most significant contaminations, is expected to dominate over the B-mode signals of the primordial gravitational waves (sometimes called as the primary B-modes). Many studies have been devoted for foreground rejection techniques [3-5] and it would be possible to remove the foreground contamination sufficiently for the detection of the primordial gravitational waves of $r \sim 0.001$ 
[6]. Even if the foreground contamination is successfully removed, there is still a significant contamination due to gravitational lensing from mass distribution between the last scattering surface and an observer. The gravitational lensing effect on the propagation of CMB photons disturbs the spatial pattern of the polarization map, which converts a small portion of E-modes into B-modes [7]. The lensing B-modes on large scales has a spectrum like white noise and their amplitude is comparable to that of the primary B-modes of $r=0.01$ on the scales of the recombination bump $(\ell \sim 10-100)$ [8]. In addition to precise measurement of the lensing B-modes, subtraction of the lensing B-modes, usually referred to as delensing, will be required in ongoing and future $\mathrm{CMB}$ experiments. It is expected to improve detection sensitivity of the primary B-modes [9-13] and even the signals of other non-lensing sources such as cosmic strings (e.g. [14, 15]), specific phenomena of modified gravity theories (e.g., [16]), and self-ordering scalar fields [17] (more generically, any cosmic defect network [18]).

For delensing, we need to know the lensing mass distribution, which is described by the lensing potential. Measurement of the lensing potential became realized recently by multiple observations of CMB polarizations such as ACTPol [19], PLANCK [20], POLARBEAR [21] and SPTpol [22, 23]. Near term and next generation CMB observations such as Advanced ACT [24], Simons Array [25], SPT-3G [26], CMB Stage-IV [27] will greatly improve sensitivity to the lensing potential. In addition, mass tracers at high redshifts such as the cosmic infrared background would be also able to be used for delensing $[12,28]$.

To perform likelihood analysis of the B-modes, we should understand and characterize statistical properties of the lensing and delensed B-modes. The lensing B-modes are known to be non-Gaussian field, and their statistical properties and the impact on the cosmological parameter constraints have been discussed in several works [29-32]. These studies showed that off-diagonal correlations of the B-mode power spectrum lead to notable degradation in the cosmological parameter estimation. On the other hand, statistical properties of the delensed B-modes, the knowledge of which is indispensable to analyses in future B-mode experiments, has not been explored in detail.

The main purpose of this paper is to explore how delensing changes the non-Gaussian properties of the lensing B-modes, in particular the off-diagonal elements of the power spectrum correlation matrix and the probability distribution function (PDF) of the power spectrum amplitude. We also discuss its impact on the estimation of the tensor-to-scalar ratio. To explore statistical properties of the delensed B-modes, we synthesize Monte Carlo samples of simulated CMB maps. Using the simulated maps, we estimate the lensing potentials and compute the delensed B-modes. The samples of the lensing and delensed B-modes are used for simulating the power spectrum covariance and the PDF of the power spectrum amplitude. To understand the simulation results, treating the delensed B-mode polarization as a weakly non-Gaussian field, we construct an analytic model which explains the simulated power spectrum covariance and also the simulated PDF of the power spectrum amplitude.

This paper is organized as follows: In Sec. 2, we describe the procedures of our delensing analysis and generation of Monte Carlo samples. In Sec. 3, we show the simulation results, focusing on the power spectrum covariance and PDF of power spectrum amplitude of the Bmode polarization. In Sec. 4, we present analytic expressions for the statistics of the delensed B-modes and compare them with the simulation results. In Sec. 5, we show the impact of the non-Gaussianity on the estimation of the tensor-to-scalar ratio. Finally, Sec. 6 is devoted to our conclusion and discussion.

Throughout this paper, we assume a flat $\Lambda$ CDM model characterized by six parameters which are the baryon density $\left(\Omega_{\mathrm{b}} h^{2}\right)$, non-relativistic matter density $\left(\Omega_{\mathrm{m}} h^{2}\right)$, dark energy 
density $\left(\Omega_{\Lambda}\right)$, scalar spectral index $\left(n_{\mathrm{s}}\right)$, scalar amplitude defined at $k=0.05 \mathrm{Mpc}^{-1}\left(A_{\mathrm{s}}\right)$, and reionization optical depth $(\tau)$. The cosmological parameters have the best-fit values of PLANCK 2013 results [33]; $\Omega_{\mathrm{b}} h^{2}=0.0220, \Omega_{\mathrm{m}} h^{2}=0.1409, \Omega_{\Lambda}=0.6964, n_{\mathrm{s}}=0.9675$, $A_{\mathrm{S}}=2.215 \times 10^{-9}$, and $\tau=0.0949$.

\section{Simulation Method}

In our analysis, to show non-Gaussian structure involved in the delensed B-mode power spectrum, we first simulate lensed CMB polarization maps, and then perform quadratic lensing reconstruction and delensing. The number of our Monte Carlo samples is 10000. In this section, we describe our method of simulating lensed polarization maps, quadratic lensing reconstruction and delensing. Note that the simulation method described below corresponds to our previous paper [34].

\subsection{Map simulation}

We denote the polarization anisotropies at a position $\hat{\boldsymbol{n}}$ on the last scattering surface as $[Q \pm \mathrm{i} U](\hat{\boldsymbol{n}})$. The lensed polarization anisotropies in the direction $\hat{\boldsymbol{n}}$, are then given by (e.g., $[7])$ :

$$
[Q \pm \mathrm{i} U](\hat{\boldsymbol{n}})=[Q \pm \mathrm{i} U](\hat{\boldsymbol{n}}+\boldsymbol{d}(\hat{\boldsymbol{n}})),
$$

where $\boldsymbol{d}$ is the deflection angle, and is given by the gradient of the lensing potential $\boldsymbol{\nabla} \phi$. Here we ignore curl modes in our simulation (see e.g. [35]). Instead of being expressed as spin-2 quantities, the following $\mathrm{E}$ and $\mathrm{B}$ mode polarizations are usually analysed in harmonic space (e.g., [7]):

$$
[E \pm \mathrm{i} B]_{\ell m}=-\int \mathrm{d} \hat{\boldsymbol{n}}_{ \pm 2} Y_{\ell m}^{*}(\hat{\boldsymbol{n}})[Q \pm \mathrm{i} U](\hat{\boldsymbol{n}}),
$$

where we denote the spin-2 spherical harmonics as ${ }_{22} Y_{\ell m}$. Similarly, with the spin-0 spherical harmonics, $Y_{\ell m}$, the lensing potential is transformed into the harmonic space as

$$
\phi_{L M}=\int \mathrm{d} \hat{\boldsymbol{n}} Y_{L M}^{*}(\hat{\boldsymbol{n}}) \phi(\hat{\boldsymbol{n}}) .
$$

To simulate the lensed CMB polarization map, we first compute the unlensed angular power spectra of the E-mode polarization $\left(C_{\ell}^{\mathrm{EE}}\right)$ and lensing potential $\left(C_{L}^{\phi \phi}\right)$ with CAMB [36]. In Lenspix [37], according to these spectra, the harmonic coefficients, $E_{\ell m}$ and $\phi_{L M}$, are generated as zero mean random Gaussian fields. Note that, in our lensing simulation, the primary B-mode polarization at the last scattering surface is not included since the lensed primary $\mathrm{B}$-mode is much smaller than the lensing B-mode. Then, $E_{\ell m}$ is transformed into Q and U maps while $\phi_{\ell m}$ is transformed into $\phi(\hat{\boldsymbol{n}})$. Finally, the $\mathrm{Q}$ and $\mathrm{U}$ maps are remapped according to Eq. (2.1) using $\phi(\hat{\boldsymbol{n}})$. In our simulation, the Healpix pixelization parameter (nside) is set to be 2048 .

Expanding Eq. (2.1) up to the first order of the lensing potential, the B modes of the lensed polarization field are described as (e.g., [38])

$$
\tilde{B}_{\ell m}=-\mathrm{i} \sum_{\ell^{\prime} m^{\prime}} \sum_{L M}\left(\begin{array}{ccc}
\ell & \ell^{\prime} & L \\
m & m^{\prime} & M
\end{array}\right) \mathcal{S}_{\ell \ell^{\prime} L}^{(-)} E_{\ell^{\prime} m^{\prime}}^{*} \phi_{L M}^{*},
$$


where we ignore the primary B-mode. The quantity $\mathcal{S}_{\ell \ell^{\prime} L}^{( \pm)}$represents the mode coupling induced by the lensing:

$$
\mathcal{S}_{\ell \ell^{\prime} L}^{( \pm)}=p^{( \pm)} \sqrt{\frac{(2 \ell+1)\left(2 \ell^{\prime}+1\right)(2 L+1)}{16 \pi}}\left[-\ell(\ell+1)+\ell^{\prime}\left(\ell^{\prime}+1\right)+L(L+1)\right]\left(\begin{array}{ccc}
\ell & \ell^{\prime} & L \\
2 & -2 & 0
\end{array}\right) .
$$

Here $p^{(+)}\left(p^{(-)}\right)$is unity if $\ell+\ell^{\prime}+L$ is an even (odd) integer and zero otherwise. Eq. (2.4) is known to be a good analytic approximation for the lensing B-modes on large scales. From Eq. (2.4), the lensing B-modes in the absence of the primary B-modes are simply expressed in terms of a convolution between the E-mode polarization and lensing potential as

$$
\widetilde{B}_{\ell m}=\mathscr{B}_{\ell m}[E, \phi],
$$

where we simplify Eq. (2.4) by defining a convolution operator for two multipole moments:

$$
\mathscr{B}_{\ell m}[\alpha, \beta] \equiv-\mathrm{i} \sum_{\ell^{\prime} m^{\prime}} \sum_{L M}\left(\begin{array}{ccc}
\ell & \ell^{\prime} & L \\
m & m^{\prime} & M
\end{array}\right) \mathcal{S}_{\ell \ell^{\prime} L}^{(-)} \alpha_{\ell^{\prime} m^{\prime}}^{*} \beta_{L M}^{*} .
$$

\subsection{Quadratic lensing reconstruction}

From the lensed polarization map, we reconstruct the lensing potential $\phi$ with the following method. Lensing induces the off-diagonal elements of the covariance matrix $\left(\ell \neq \ell^{\prime}\right.$ or $\left.m \neq m^{\prime}\right)$ between two lensed polarization anisotropies $(X, Y=E, B)$ as

$$
\left\langle\widetilde{X}_{\ell m} \widetilde{Y}_{\ell^{\prime} m^{\prime}}\right\rangle_{\mathrm{CMB}}=\sum_{L, M}\left(\begin{array}{ccc}
\ell & \ell^{\prime} & L \\
m & m^{\prime} & M
\end{array}\right) f_{\ell \ell^{\prime} L}^{\mathrm{XY}} \phi_{L M}^{*}+\mathcal{O}\left(\phi^{2}\right),
$$

where the operation $\langle\cdots\rangle_{\mathrm{CMB}}$ denotes the ensemble average over the primary CMB anisotropies. The weight function $f_{\ell \ell^{\prime} L}^{\mathrm{XY}}$ in Eq. (2.8) is defined as [39]

$$
\begin{aligned}
f_{\ell \ell^{\prime} L}^{\mathrm{EE}} & =\mathcal{S}_{\ell \ell^{\prime} L}^{(+)} C_{\ell^{\prime}}^{\mathrm{EE}}+\mathcal{S}_{\ell^{\prime} \ell L}^{(+)} C_{\ell}^{\mathrm{EE}}, \\
f_{\ell \ell^{\prime} L}^{\mathrm{EB}} & =\mathrm{i} \mathcal{S}_{\ell^{\prime} \ell L}^{(-)} C_{\ell}^{\mathrm{EE}} .
\end{aligned}
$$

Eq. (2.8) motivates the following form for a quadratic lensing estimator (see e.g., [39]):

$$
\left[\widehat{\phi}_{L M}^{\mathrm{XY}}\right]^{*}=A_{L}^{\mathrm{XY}} \sum_{\ell \ell^{\prime}} \sum_{m m^{\prime}}\left(\begin{array}{ccc}
\ell & \ell^{\prime} & L \\
m & m^{\prime} & M
\end{array}\right) \frac{1}{\Delta \mathrm{XY}}\left[f_{\ell \ell^{\prime} L}^{\mathrm{XY}}\right]^{*} \frac{\widehat{X}_{\ell m}}{\widehat{C}_{\ell}^{\mathrm{XX}}} \frac{\widehat{Y}_{\ell^{\prime} m^{\prime}}}{\widehat{C}_{\ell^{\prime}}^{\mathrm{YY}}},
$$

where $\widehat{X}$ and $\widehat{Y}$ are observed polarization anisotropies. The quantities, $\widehat{C}_{\ell}^{\mathrm{XX}}$ and $\widehat{C}_{\ell}^{\mathrm{YY}}$, are their angular power spectra. $\Delta^{\mathrm{XY}}$ is 2 for $\mathrm{XY}=\mathrm{EE}$ and 1 for $\mathrm{XY}=\mathrm{EB}$. The quantity $A_{L}^{\mathrm{XY}}$ is given by

$$
A_{L}^{\mathrm{XY}}=\left\{\frac{1}{2 L+1} \sum_{\ell \ell^{\prime}} \frac{\left[f_{\ell \ell^{\prime} L}^{\mathrm{XY}}\right]^{*} f_{\ell \ell^{\prime} L}^{\mathrm{XY}}}{\Delta^{\mathrm{XY}} \widehat{C}_{\ell}^{\mathrm{XX}} \widehat{C}_{\ell^{\prime}}^{\mathrm{YY}}}\right\}^{-1} .
$$

Note that, to mitigate biases coming from the higher order terms of $\phi$, we use the lensed power spectrum $\left(\widetilde{C}_{\ell}^{\mathrm{EE}}\right)$ in Eq. $(2.10)[40,41]$ instead of the unlensed power spectrum. The 
optimal estimator of the lensing potential is then obtained as a linear combination of the EE and EB quadratic estimators:

$$
\widehat{\phi}_{L M}=A_{L}^{\phi}\left(\frac{1}{A_{L}^{\mathrm{EE}}} \widehat{\phi}_{L M}^{\mathrm{EE}}+\frac{1}{A_{L}^{\mathrm{EB}}} \widehat{\phi}_{L M}^{\mathrm{EB}}\right)
$$

with

$$
A_{L}^{\phi} \equiv \frac{1}{\left(A_{L}^{\mathrm{EE}}\right)^{-1}+\left(A_{L}^{\mathrm{EB}}\right)^{-1}}
$$

In lensing reconstruction, we add a random white noise with a Gaussian beam factor whose power spectrum is described as

$$
N_{\ell}^{\mathrm{P}} \equiv\left(\frac{\Delta_{\mathrm{P}}}{T_{\mathrm{CMB}}}\right)^{2} \exp \left[\frac{\ell(\ell+1) \theta^{2}}{8 \ln 2}\right] .
$$

Here $T_{\mathrm{CMB}}=2.7 \mathrm{~K}$ is the mean temperature of CMB, $\theta$ is a beam size, and $\Delta_{\mathrm{P}}$ is a noise level of polarization measurement. The multipole $\ell$ is an integer between 2 and 2000 . The fiducial values for the experiment assumed in our analysis are $\Delta_{\mathrm{P}}=6 \mu \mathrm{K}$-arcmin and $\theta=4$ arcmin. In such a situation, quadratic lensing reconstruction described here is a nearly optimal method [42]. We estimate the lensing potential, using the $\mathrm{E}$ and B-modes up to $\ell=2000$. Note that these values are similar to those of the Simons Array [25] which is expected to cover a wide range of the entire sky. Advanced ACT also has a similar instrumental specification [24].

\subsection{Quadratic delensing}

Once we obtain the lensing potential using the quadratic estimator, we can estimate the lensing B-modes in a similar manner of Eq. (2.6). On large scales, the lensing B-mode is well estimated by [10]:

$$
\mathscr{B}_{\ell m}\left[\widehat{E}^{\mathrm{w}}, \widehat{\phi}^{\mathrm{w}}\right],
$$

where, again, the operator $\mathscr{B}_{\ell m}$ convolves two fields in the same way that it forms the lensing B-modes from the E-modes and lensing potential. We denote $\widehat{E}_{\ell m}^{\mathrm{w}}$ and $\widehat{\phi}_{\ell m}^{\mathrm{w}}$ as the Wiener filtered multipoles, i.e., the measured E-mode polarization and lensing potential multiplied by the corresponding Wiener filters $\left(W^{\mathrm{E}}\right.$ and $\left.W^{\phi}\right)$ defined as $[9,10]$

$$
\begin{aligned}
W_{\ell}^{\mathrm{E}} & =\frac{C_{\ell}^{\mathrm{EE}}}{C_{\ell}^{\mathrm{EE}}+N_{\ell}^{\mathrm{P}}}, \\
W_{\ell}^{\phi} & =\frac{C_{\ell}^{\phi \phi}}{C_{\ell}^{\phi \phi}+A_{\ell}^{\phi}} .
\end{aligned}
$$

The residual field of the B-mode polarization after delensing $\left(B_{\ell m}^{\mathrm{d}}\right)$ is then evaluated as [10]

$$
B_{\ell m}^{\mathrm{d}}=\widehat{B}_{\ell m}-\mathscr{B}_{\ell m}\left[\widehat{E}^{\mathrm{w}}, \widehat{\phi}^{\mathrm{w}}\right] .
$$

To avoid delensing bias (see e.g. appendix A of Ref. [34]), only the multipoles between $\ell=301$ and 2000 are taken into account in the calculation of lensing reconstruction. Then, we evaluate the delensed B-mode polarization only between $\ell=2$ and 300. Note that, as shown in our previous paper [34], the filtering of large scale multipoles in lensing reconstruction is required also for an unbiased estimate of the lensing potential in presence of $1 / f$ noises. 

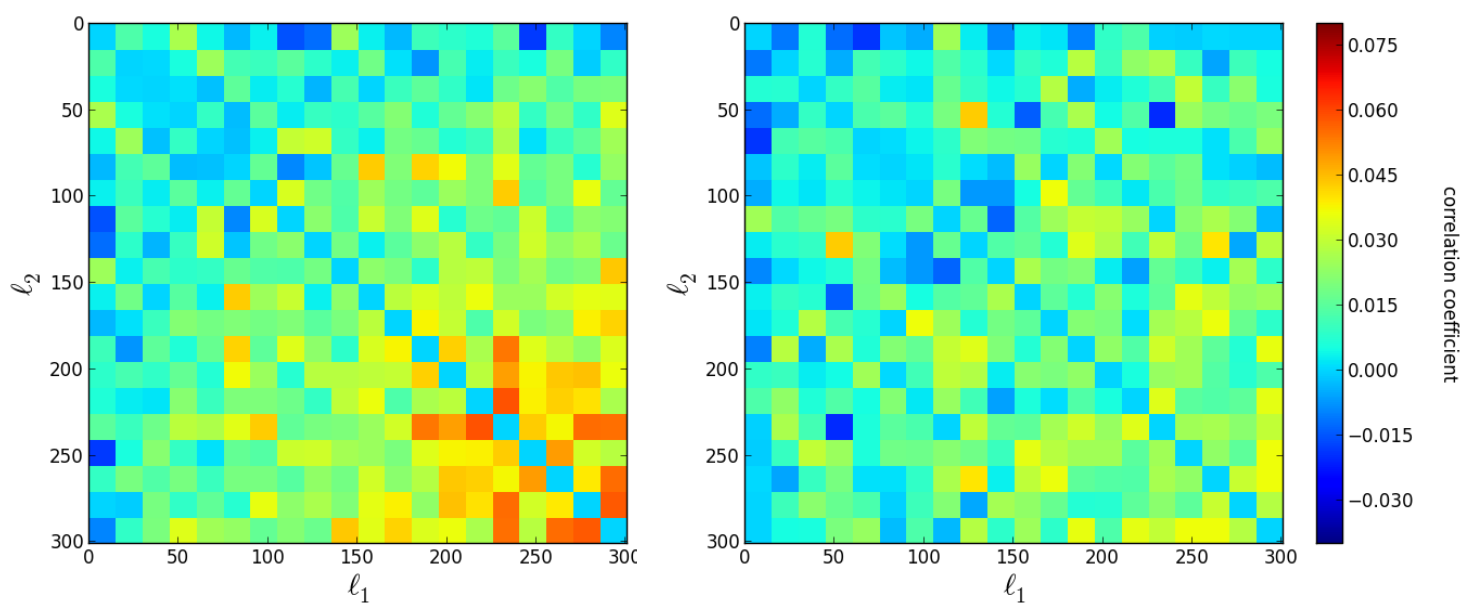

Figure 1. Correlation coefficients of the angular power spectrum of the lensing (Left) and delensed (Right) B-modes between different multipoles defined in Eq. (3.4). The correlation coefficients are computed from 10000 realizations of the lensed and delensed QU maps. We apply flat binning. The bin width is $\Delta \ell=15$ and the multipoles between $\ell=1$ and 300 are divided into 20 bins. Note that the diagonal elements are removed for an illustrative purpose.

\section{Non-Gaussian Signature of Delensed B-mode Polarization}

\subsection{Power spectrum covariance}

Significance of non-Gaussian properties of the B-mode polarization can be seen in the power spectrum covariance. In the case of a Gaussian field, the power spectra at different multipoles are uncorrelated, and the covariance matrix has only diagonal elements. On the other hand, a non-Gaussian field such as the lensing B-modes has off-diagonal elements of notable magnitude in the covariance matrix as shown in the previous studies [29-32]. Here, we present the simulated covariance matrix of the delensed B-mode power spectrum and compare it with that of the lensing B-mode power spectrum. Note that, to see non-Gaussian structure of the delensed B-modes, we do not include the tensor perturbations at first. The case including the tensor perturbations is discussed in Sec. 5 .

To compute the power spectrum covariance, the multipole is binned into 20 bins. The binned angular power spectrum at $i$ th multipole bin is defined as

$$
\widehat{C}_{i}=\frac{1}{N_{i}} \sum_{\ell \in\left[\ell_{i-1}, \ell_{i}-1\right]} \widehat{C}_{\ell},
$$

where $N_{i}$ is the number of multipoles in $i$ th bin, and we choose $\ell_{i}=15 i+1$. For the first bin $(i=1)$, we remove $\ell=1$. The angular power spectrum is computed with the usual method:

$$
\widehat{C}_{\ell}=\frac{1}{2 \ell+1} \sum_{m=-\ell}^{\ell}\left|B_{\ell m}\right|^{2} .
$$

The power spectrum covariance between each multipole bin $i$ and $j$ is then evaluated as

$$
\operatorname{Cov}_{i j}=\left\langle\widehat{C}_{i} \widehat{C}_{j}\right\rangle-\left\langle\widehat{C}_{i}\right\rangle\left\langle\widehat{C}_{j}\right\rangle .
$$



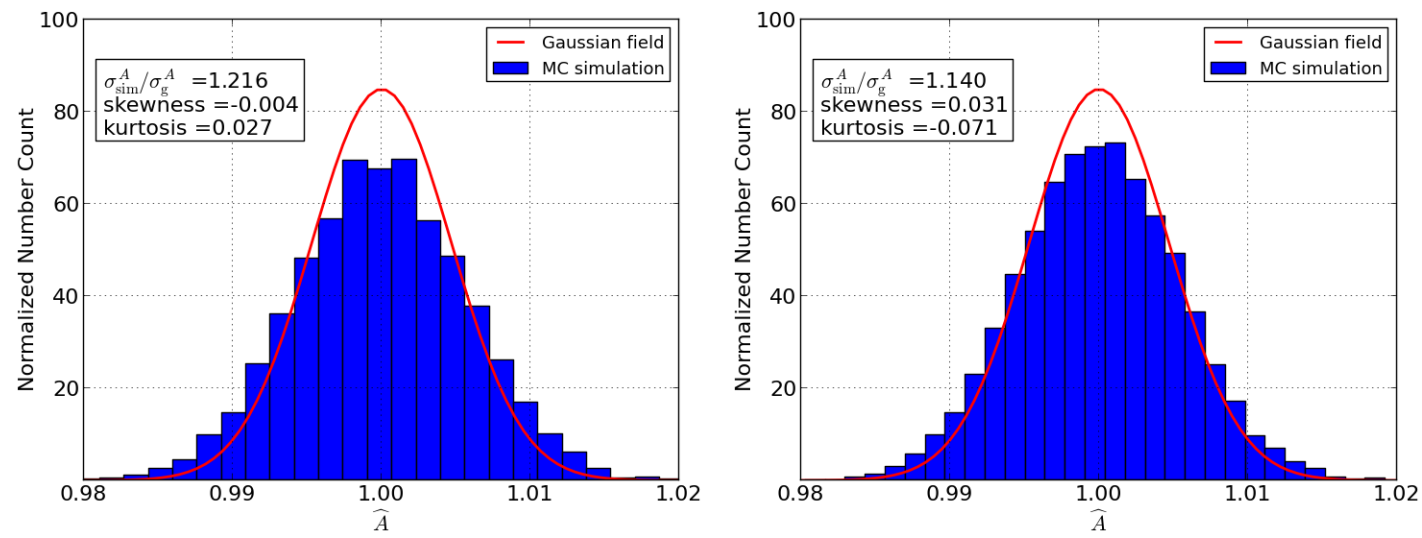

Figure 2. Histogram of the estimated power spectrum amplitude of the lensing (Left) and delensed (Right) B-modes. The number of the samples is 10000. The solid line shows the PDF obtained on the assumption that the lensing/delensed B-modes obey Gaussian statistics. We show the ratio of the standard deviation computed from the simulation $\left(\sigma_{\text {sim }}^{A}\right)$ to that in the case of a Gaussian CMB field $\left(\sigma_{\mathrm{g}}^{A}\right)$. The skewness and kurtosis of the histogram are also shown. The fiducial power spectrum is evaluated as the sample mean of the simulated power spectra.

$\langle\cdots\rangle$ is the sample mean of the operand.

In Fig. 1, we show the correlation coefficients of the lensing (Left) and delensed (Right) B-mode power spectrum defined as [32]

$$
R_{i j}=\frac{\operatorname{Cov}_{i j}}{\sqrt{\operatorname{Cov}_{i i} \operatorname{Cov}_{j j}}} .
$$

The values of the off-diagonal elements increase at higher multipoles. The typical values of the correlation coefficients of the lensing B-modes are $\sim 6-8 \%$ at $\ell \sim 200-300$, which is consistent with the previous results obtained in Ref. [32] ${ }^{1}$. On the other hand, we can confirm that the off-diagonal elements of the correlation matrix of the delensed B-modes are definitely smaller than those of the lensing B-modes. These results imply that the delensing operation reduced the off-diagonal covariance more efficiently than the diagonal covariance.

\subsection{Probability distribution function of power spectrum amplitude}

To discuss the non-Gaussian covariance more quantitatively, next we consider the estimation of the power spectrum amplitude. In the case of a Gaussian field, the optimal estimator of the power spectrum amplitude is given by [43]

$$
\widehat{A}=\frac{\sum_{\ell}(2 \ell+1) \widehat{A}_{\ell}}{\sum_{\ell}(2 \ell+1)}
$$

where $\widehat{A}_{\ell}$ is the amplitude parameter at each multipole:

$$
\widehat{A}_{\ell}=\frac{\widehat{C}_{\ell}}{C_{\ell}}
$$

\footnotetext{
${ }^{1}$ Note that Ref. [32] showed the correlation coefficients with 2 times wider bins in which the off-diagonal elements are apparently larger than those in Fig. 1.
} 
The angular power spectrum in the denominator $\left(C_{\ell}\right)$ is the fiducial power spectrum defined as the ensemble average of $\widehat{C}_{\ell}$. The parameter $\widehat{A}_{\ell}$ is a measured amplitude relative to $C_{\ell}$ at each multipole. In the case of a Gaussian CMB field, the expected variance of $\widehat{A}$ is given by

$$
\left(\sigma_{\mathrm{g}}^{A}\right)^{2}=\frac{2}{\sum_{\ell}(2 \ell+1)} .
$$

Deviation of the simulated variance from $\left(\sigma_{\mathrm{g}}^{A}\right)^{2}$ indicates a non-Gaussian signature.

The histograms of the estimator $\widehat{A}(3.5)$ of the lensing and delensed B-modes are shown in Fig. 2. They are synthesized from 10000 realizations. In the estimation of $\widehat{A}$, we use the B-modes between $\ell=2$ and 300. We evaluate the fiducial power spectrum as the sample mean of the simulated 10000 power spectra. Consequently, the sample mean of $\widehat{A}$ is unbiased. The standard deviation of $\widehat{A}\left(\sigma_{\mathrm{sim}}^{A}\right)$ divided by that in the case of a Gaussian field $\left(\sigma_{\mathrm{g}}^{A}\right)$ is shown in the figure.

Before delensing, the standard deviation of the estimator is $\sim 22 \%$ larger than that in the case of a Gaussian CMB field. On the other hand, after delensing, the discrepancy is decreased to $\sim 15 \%$, which is considered to be an outcome of the decrease of the off-diagonal correlations. Note that, even in the case that the lensing/delensed B-modes are assumed to be Gaussian, the skewness and kurtosis of the amplitude estimator have non-zero values because the amplitude estimator is quadratic in the CMB field. In our case, the skewness and kurtosis of the estimator are 0.0057 and $1.3 \times 10^{-7}$, respectively. The skewness and kurtosis are also shown, and they deviate from those in the case of a Gaussian CMB field within only $\leq 0.03$ for the skewness and $\leq 0.07$ for the kurtosis, respectively.

\section{Modeling Statistical Properties of Delensed B-mode}

To understand the statistical properties of the delensed B-modes shown in the previous section, we construct an analytic model of the PDF of the lensing and delensed B-modes which is consistent with the simulation results. The simulation results suggest that the deviation from a Gaussian field is appeared primarily as the small increase of the variance $\left(\sigma^{A}\right)^{2}$. Motivated by this fact, we try to express the PDF of the lensing and delensed B-modes as a perturbed Gaussian distribution. The Edgeworth expansion, which assumes a weakly non-Gaussian PDF, is used for such purpose. We derive a PDF for the lensing and delensed B-modes which includes corrections up to 4th order of the Edgeworth expansion.

\subsection{Probability distribution function for weakly non-Gaussian fields}

We denote $n_{L}\left(=\sum_{\ell}(2 \ell+1)\right)$ independent Gaussian random variables as $a_{\ell m}$. The joint PDF of $\boldsymbol{a}=\left\{a_{\ell m}\right\}$ is given by

$$
P_{\mathrm{g}}(\boldsymbol{a})=\frac{1}{(2 \pi)^{n_{L} / 2}|\mathbf{C}|^{1 / 2}} \exp \left[-\frac{1}{2} \boldsymbol{a}^{t} \mathbf{C}^{-1} \boldsymbol{a}\right] .
$$

Here, we denote the covariance of $a_{\ell m} \mathrm{~s}$ as $\mathbf{C}=\left\langle\boldsymbol{a} \boldsymbol{a}^{t}\right\rangle$. The PDF including the corrections from the Edgeworth expansion at 4th order is given by

$$
P(\boldsymbol{a})=[1+k(\boldsymbol{a})] P_{\mathrm{g}}(\boldsymbol{a}),
$$


where the 4th-order correction term $k(\boldsymbol{a})$ is described as (see e.g. [44])

$$
\begin{aligned}
k(\boldsymbol{a})= & \frac{1}{24 P_{\mathrm{g}}(\boldsymbol{a})} \sum_{\ell_{i} m_{i}} T_{m_{1} m_{2} m_{3} m_{4}}^{\ell_{1} \ell_{2} \ell_{3} \ell_{4}} \frac{\partial}{\partial a_{\ell_{1} m_{1}}} \frac{\partial}{\partial a_{\ell_{2} m_{2}}} \frac{\partial}{\partial a_{\ell_{3} m_{3}}} \frac{\partial}{\partial a_{\ell_{4} m_{4}}} P_{\mathrm{g}}(\boldsymbol{a}) \\
= & \frac{1}{24} \sum_{\ell_{i} m_{i}} T_{m_{1} m_{2} m_{3} m_{4}}^{\ell_{1} \ell_{2} \ell_{3} \ell_{4}}\left(\bar{a}_{\ell_{1} m_{1}} \bar{a}_{\ell_{2} m_{2}} \bar{a}_{\ell_{3} m_{3}} \bar{a}_{\ell_{4} m_{4}}\right. \\
& \left.-6 \mathbf{C}_{\ell_{1} m_{1}, \ell_{2} m_{2}}^{-1} \bar{a}_{\ell_{3} m_{3}} \bar{a}_{\ell_{4} m_{4}}+3 \mathbf{C}_{\ell_{1} m_{1}, \ell_{2} m_{2}}^{-1} \mathbf{C}_{\ell_{3} m_{3}, \ell_{4} m_{4}}^{-1}\right) .
\end{aligned}
$$

The quantity $\bar{a}_{\ell m}=\left[\mathbf{C}^{-1} \boldsymbol{a}\right]_{\ell m}$ is the inverse-variance filtered multipole. We also denote the connected part of the four-point correlation as

$$
T_{m_{1} m_{2} m_{3} m_{4}}^{\ell_{1} \ell_{2} \ell_{3} \ell_{4}}=\left\langle a_{\ell_{1} m_{1}} a_{\ell_{2} m_{2}} a_{\ell_{3} m_{3}} a_{\ell_{4} m_{4}}\right\rangle_{c}
$$

We assume that the covariance matrix of $a_{\ell m}$ is diagonal:

$$
\mathbf{C}_{\ell m, \ell^{\prime} m^{\prime}}=\delta_{\ell \ell^{\prime}} \delta_{m,-m^{\prime}}(-1)^{m} C_{\ell}
$$

This simplifies the PDF as

$$
P(\boldsymbol{a})=\frac{1+k(\boldsymbol{a})}{(2 \pi)^{n_{L} / 2}} \prod_{\ell} C_{\ell}^{-\frac{2 \ell+1}{2}} \exp \left[-\sum_{m=-\ell}^{\ell} \frac{\left|a_{\ell m}\right|^{2}}{2 C_{\ell}}\right] .
$$

Note that the PDF described above is used to derive the optimal trispectrum estimator of the lensing potential power spectrum $[45,46]$.

In the above PDF, we ignore the third order term in the Edgeworth expansion which is expressed in terms of the B-mode bispectrum because the bispectrum of the B-mode polarization has the odd-parity symmetry and is not generated in the standard cosmology.

Let us discuss the statistical property of the power spectrum amplitude, $\widehat{A}$. Using the PDF $P(\boldsymbol{a})$ given by Eq. (4.7), we obtain the mean of the estimator as follows:

$$
\langle\widehat{A}\rangle=\langle(\widehat{A}+\widehat{A} k(\boldsymbol{a}))\rangle_{\mathrm{g}}=1 .
$$

Here, the operation $\langle\cdots\rangle$ means averaging based on the whole PDF $P(\boldsymbol{a})$, while the operation $\langle\cdots\rangle_{\mathrm{g}}$ means that on the Gaussian PDF $P_{\mathrm{g}}(\boldsymbol{a})$. The derivation is shown in appendix A.1. On the other hand, from the PDF described in Eq. (4.7), the variance of the amplitude estimator is given by (see appendix A.2)

$$
\left(\sigma^{A}\right)^{2} \equiv\left\langle\widehat{A}^{2}\right\rangle-1=\frac{\left(\sigma_{\mathrm{g}}^{A}\right)^{2}}{n_{L}} \sum_{\ell \ell^{\prime}} \frac{(2 \ell+1)\left(2 \ell^{\prime}+1\right)}{2} \frac{\operatorname{Cov}_{\ell \ell^{\prime}}}{C_{\ell} C_{\ell^{\prime}}},
$$

where the power spectrum covariance, $\operatorname{Cov}_{\ell \ell^{\prime}}$, is expressed as

$$
\operatorname{Cov}_{\ell \ell^{\prime}}=\frac{2 C_{\ell} C_{\ell^{\prime}}}{2 \ell+1} \delta_{\ell \ell^{\prime}}+\frac{1}{(2 \ell+1)\left(2 \ell^{\prime}+1\right)} \sum_{m m^{\prime}}(-1)^{m+m^{\prime}} T_{m,-m, m^{\prime},-m^{\prime}}^{\ell \ell \ell^{\prime} \ell^{\prime}}
$$

The PDF of the power spectrum amplitude $\widehat{A}$ is given as the chi square distribution with a correction term from the kurtosis (see appendix A.3 for derivation):

$$
P(\widehat{A})=\frac{\left(n_{L} / 2\right)^{n_{L} / 2}}{\Gamma\left(n_{L} / 2\right)} \widehat{A}^{\frac{n_{L}}{2}-1} \mathrm{e}^{-\frac{n_{L}}{2} \widehat{A}}\left\{1+n_{L} \frac{\left(\sigma^{A}\right)^{2}-\left(\sigma_{\mathrm{g}}^{A}\right)^{2}}{4\left(\sigma_{\mathrm{g}}^{A}\right)^{2}}\left[\frac{n_{L}}{n_{L}+2} \widehat{A}^{2}-2 \widehat{A}+1\right]\right\} .
$$

Once we obtain the power spectrum covariance, we can actually evaluate the variance of the power spectrum amplitude and the PDF. 


\subsection{Expression for B-mode power spectrum covariance}

Next, we discuss mathematical expression for the B-mode power spectrum covariance. At first, we summarize the derivation of the analytic expression for the power spectrum covariance of the lensing B-modes developed by Refs. [29-32]. The lensing B-mode power spectrum on large scales $(\ell \lesssim 300)$ is well described by a convolution of the E-mode polarization and lensing potential power spectra (see e.g., [10]):

$$
\widetilde{C}_{\ell}^{\mathrm{BB}}=\frac{1}{2 \ell+1} \sum_{\ell^{\prime} L}\left(\mathcal{S}_{\ell \ell^{\prime} L}^{(-)}\right)^{2} C_{\ell^{\prime}}^{\mathrm{EE}} C_{L}^{\phi \phi} \equiv \Xi_{\ell}\left[C^{\mathrm{EE}}, C^{\phi \phi}\right]
$$

where $S_{\ell \ell^{\prime} L}^{(-)}$is given in Eq. (2.5), and we define a convolution operator:

$$
\Xi_{\ell}[A, B]=\frac{1}{2 \ell+1} \sum_{\ell_{1} \ell_{2}}\left(\mathcal{S}_{\ell \ell_{1} \ell_{2}}^{(-)}\right)^{2} A_{\ell_{1}} B_{\ell_{2}} .
$$

The fluctuations of the E-mode and lensing potential power spectra contribute to the fluctuation of the lensing B-mode power spectrum as follows [32]:

$$
\delta \widetilde{C}_{\ell}^{\mathrm{BB}}=\sum_{\ell^{\prime}} \frac{\partial \widetilde{C}_{\ell}^{\mathrm{BB}}}{\partial C_{\ell^{\prime}}^{\mathrm{EE}}} \delta C_{\ell^{\prime}}^{\mathrm{EE}}+\sum_{\ell^{\prime}} \frac{\partial \widetilde{C}_{\ell}^{\mathrm{BB}}}{\partial C_{\ell^{\prime}}^{\phi \phi}} \delta C_{\ell^{\prime}}^{\phi \phi} .
$$

The connected part of the covariance due to this contribution $\left\langle\widetilde{C}_{\ell}^{\mathrm{BB}} \widetilde{C}_{\ell^{\prime}}^{\mathrm{BB}}\right\rangle_{\mathrm{c}}$ is approximately given by

$$
\left\langle\widetilde{C}_{\ell}^{\mathrm{BB}} \widetilde{C}_{\ell^{\prime}}^{\mathrm{BB}}\right\rangle_{\mathrm{c}}=\sum_{L} \frac{\partial \widetilde{C}_{\ell}^{\mathrm{BB}}}{\partial C_{L}^{\mathrm{EE}}} \frac{2\left(C_{L}^{\mathrm{EE}}\right)^{2}}{2 L+1} \frac{\partial \widetilde{C}_{\ell^{\prime}}^{\mathrm{BB}}}{\partial C_{L}^{\mathrm{EE}}}+\sum_{L} \frac{\partial \widetilde{C}_{\ell}^{\mathrm{BB}}}{\partial C_{L}^{\phi \phi}} \frac{2\left(C_{L}^{\phi \phi}\right)^{2}}{2 L+1} \frac{\partial \widetilde{C}_{\ell^{\prime}}^{\mathrm{BB}}}{\partial C_{L}^{\phi \phi}} .
$$

Here we use

$$
\left\langle\delta C_{\ell}^{X} \delta C_{\ell^{\prime}}^{X}\right\rangle=\frac{2 C_{\ell}^{X} C_{\ell^{\prime}}^{X}}{2 \ell+1} \delta_{\ell \ell^{\prime}},
$$

where $X$ is $E E$ or $\phi \phi$. Note that the power spectrum covariance also contains a fully connected term which is not expressed like the r.h.s. of Eq. (4.15), but it has only negligible contribution [31]. The approximate expression for the power spectrum covariance is then described as [32]

$$
\begin{aligned}
\operatorname{Cov}_{\ell \ell^{\prime}}^{\mathrm{BB}} & \equiv\left\langle\widetilde{C}_{\ell}^{\mathrm{BB}} \widetilde{C}_{\ell^{\prime}}^{\mathrm{BB}}\right\rangle-\left\langle\widetilde{C}_{\ell}^{\mathrm{BB}}\right\rangle\left\langle\widetilde{C}_{\ell^{\prime}}^{\mathrm{BB}}\right\rangle \\
& \simeq \frac{2\left(\widetilde{C}_{\ell}^{\mathrm{BB}}\right)^{2}}{2 \ell+1} \delta_{\ell \ell^{\prime}}+\sum_{L}\left[\frac{\partial \widetilde{C}_{\ell}^{\mathrm{BB}}}{\partial C_{L}^{\mathrm{EE}}} \frac{2\left(C_{L}^{\mathrm{EE}}\right)^{2}}{2 L+1} \frac{\partial \widetilde{C}_{\ell^{\prime}}^{\mathrm{BB}}}{\partial C_{L}^{\mathrm{EE}}}+\frac{\partial \widetilde{C}_{\ell}^{\mathrm{BB}}}{\partial C_{L}^{\phi \phi}} \frac{2\left(C_{L}^{\phi \phi}\right)^{2}}{2 L+1} \frac{\partial \widetilde{C}_{\ell^{\prime}}^{\mathrm{BB}}}{\partial C_{L}^{\phi \phi}}\right] .
\end{aligned}
$$

We now apply the above discussion to derive the power spectrum covariance for the delensed B-modes. Assuming that the E-modes are nearly cosmic-variance limited, and using the similar procedure described above, we obtain the following expression as

$$
\operatorname{Cov}_{\ell \ell^{\prime}}^{\mathrm{BB}, \mathrm{d}}=\frac{2\left(C_{\ell}^{\mathrm{BB}, \mathrm{d}}\right)^{2}}{2 \ell+1} \delta_{\ell \ell^{\prime}}+\sum_{L}\left[\frac{\partial C_{\ell}^{\mathrm{BB}, \mathrm{d}}}{\partial C_{L}^{\mathrm{EE}}} \frac{2\left(C_{L}^{\mathrm{EE}}\right)^{2}}{2 L+1} \frac{\partial C_{\ell^{\prime}}^{\mathrm{BB}, \mathrm{d}}}{\partial C_{L}^{\mathrm{EE}}}+\frac{\partial C_{\ell}^{\mathrm{BB}, \mathrm{d}}}{\partial C_{L}^{\phi \phi}} \frac{2\left(C_{L}^{\phi \phi}\right)^{2}}{2 L+1} \frac{\partial C_{\ell^{\prime}}^{\mathrm{BB}, \mathrm{d}}}{\partial C_{L}^{\phi \phi}}\right] .
$$

The actual derivation requires cumbersome calculation and is shown in appendix B.1. 

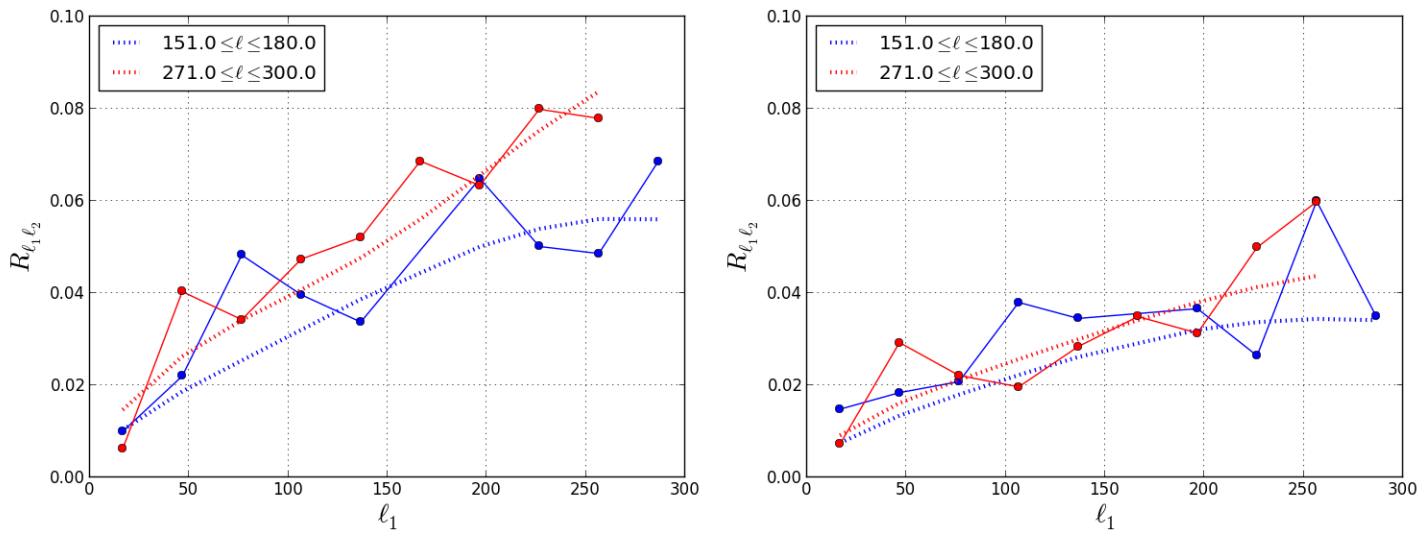

Figure 3. Correlation coefficients of the lensing (Left) and delensed (Right) B-mode power spectrum. The correlation coefficients obtained from the Monte Carlo simulation (solid) are compared with those of the analytic model (dashed). For an illustrative purpose, the multipoles up to 300 are binned into 12 bins.
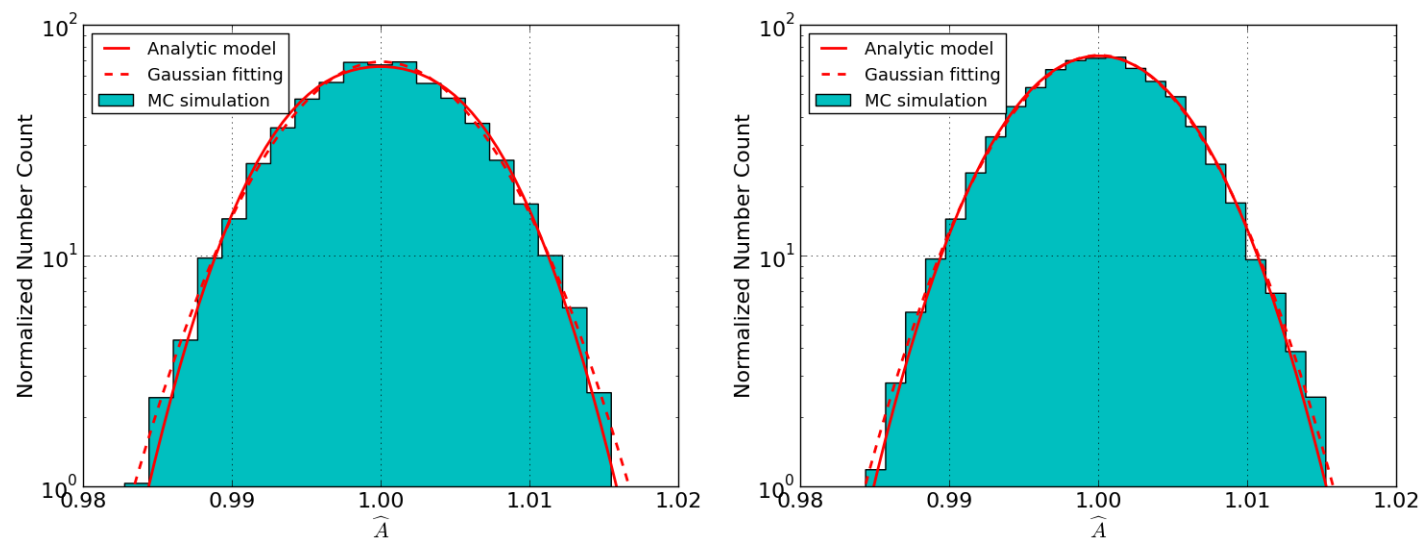

Figure 4. Theoretical PDF of the lensing (Left) and delensed (Right) B-mode power spectrum amplitude compared with the histogram shown in Fig. 2. We show two theoretical models; the analytic model of the PDF given by Eq. (4.11) (solid), and the empirical PDF obtained by fitting a normal distribution with the histogram (dashed). Note that, the y-axis is in logarithmic scale for the clarification of the effect on the variance.

\subsection{Comparison with simulation results}

Now we compare the model calculations with the results of the Monte Carlo simulation.

The correlation coefficients of the lensing and delensed B-mode power spectra are shown in Fig. 3. The simulated correlation coefficients are compared with those of the analytic models described in the previous subsection. The analytic models capture the behavior of the simulated correlation coefficients.

The analytic PDFs of the power spectrum amplitude described in Sec. 4.1 are compared with the histograms obtained from the Monte Carlo simulation in Fig. 4. Our analytic 

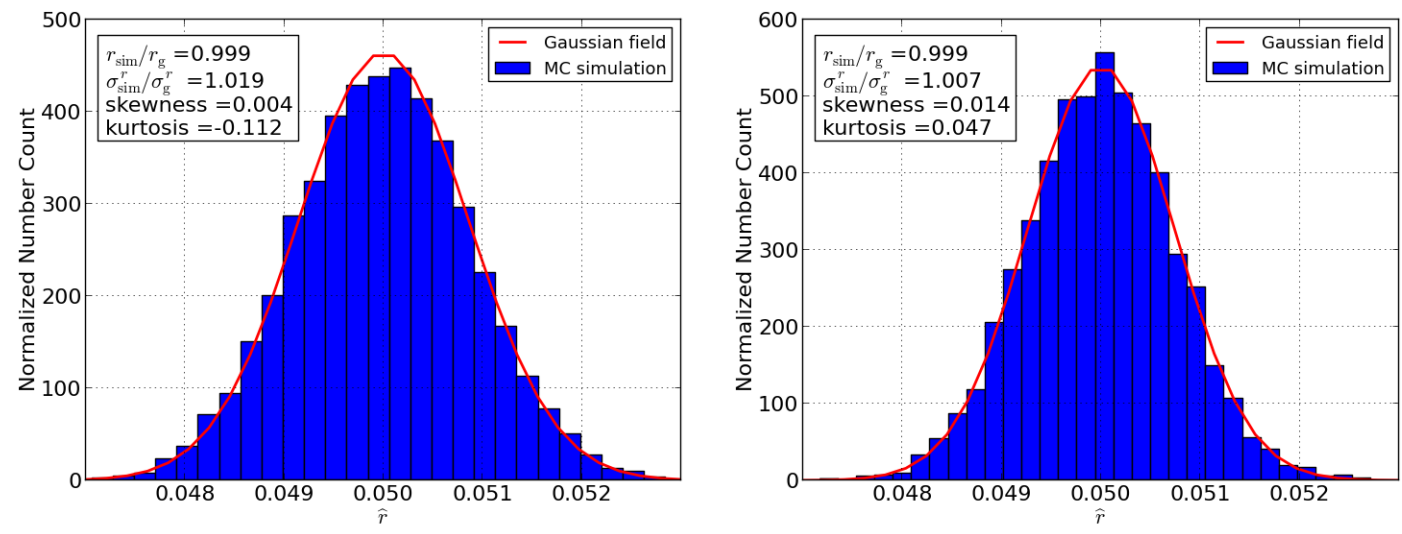

Figure 5. Histogram of the tensor-to-scalar ratio estimated from the tensor plus lensing (Left) and tensor plus delensed (Right) B-modes. The number of the samples is 10000 . The input value of the tensor-to-scalar ratio is $r=0.05$. The solid line shows the PDF in the case of a Gaussian B-mode. The fiducial power spectrum is evaluated as the sample mean of the simulated power spectra.

model of the delensed B-modes well describes the statistics of the simulated samples. The simulated standard deviation of the amplitude parameter is $\sigma^{A}=0.005357$. By use of the analytic formula, the standard deviation is estimated as $\sigma^{A}=0.005264$ which is in agreement with the simulation result within $1.8 \%$. We also show the normal distributions fitted with the histograms. Contrary to the PDFs in the case of a Gaussian field (Fig. 2), the normal distributions with the fitted variances well capture the behaviors of the simulated histograms, which also validates the use of the 4th-order Edgeworth expansion.

\section{Impact on Estimation of Tensor-to-Scalar Ratio}

Here we demonstrate the effect of non-Gaussianity from the lensing/delensed B-modes to the estimation of the tensor-to-scalar ratio $r$. At a single multipole $\ell$, the tensor-to-scalar ratio which maximizes the likelihood function is obtained by, e.g., differentiating Eq. (3) of [6] in terms of $r$ :

$$
\widehat{r}_{\ell}=\frac{\widehat{C}_{\ell}^{\mathrm{BB}}-C_{\ell}^{\mathrm{L}}}{C_{\ell}^{\mathrm{T}}},
$$

where the quantities $\widehat{C}_{\ell}^{\mathrm{BB}}, C_{\ell}^{\mathrm{L}}$ and $C_{\ell}^{\mathrm{T}}$ are the observed, lensing/delensed and tensor B-mode power spectrum, respectively. Here the spectrum $C_{\ell}^{\mathrm{T}}$ is for $r=1$. Under the assumption that the covariance of the observed B-mode power spectrum is diagonal, the expected variance of the above quantity is given by

$$
\sigma_{\ell}^{2}=\frac{2}{2 \ell+1}\left(\frac{C_{\ell}^{\mathrm{L}}+r C_{\ell}^{\mathrm{T}}}{C_{\ell}^{\mathrm{T}}}\right)^{2} .
$$

This leads to an optimal estimator of $r$ as

$$
\widehat{r}=\left(\sigma_{\mathrm{g}}^{r}\right)^{2} \sum_{\ell} \frac{\widehat{r}_{\ell}}{\sigma_{\ell}^{2}},
$$




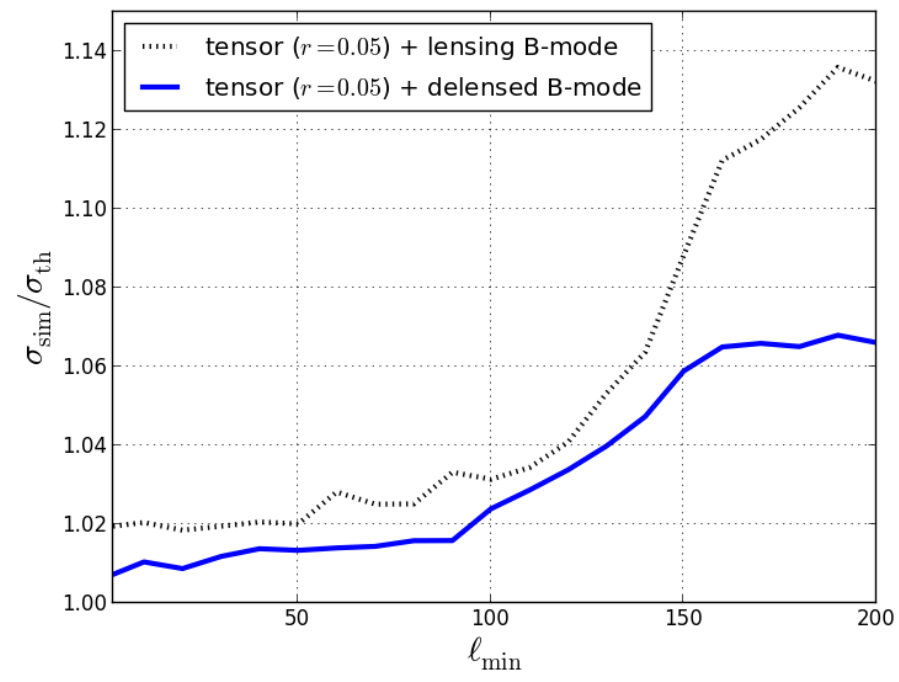

Figure 6. Standard deviations of the tensor-to-scalar ratio estimator divided by that in the case of a Gaussian B-mode $\left(\sigma_{\mathrm{sim}}^{r} / \sigma_{\mathrm{g}}^{r}\right)$ as a function of the minimum multipole included in the estimation $\left(\ell_{\mathrm{min}}\right)$ for the tensor plus lensing (dashed black) and tensor plus delensed (solid blue) B-modes. The input value of the tensor-to-scalar ratio is 0.05 . The maximum multipole in the estimation is fixed to 300 .

where $\left(\sigma_{\mathrm{g}}^{r}\right)^{2}$ is the expected variance of $\widehat{r}$ :

$$
\left(\sigma_{\mathrm{g}}^{r}\right)^{2} \equiv \frac{1}{\sum_{\ell} \sigma_{\ell}^{-2}}=\left[\sum_{\ell} \frac{2 \ell+1}{2}\left(\frac{C_{\ell}^{\mathrm{T}}}{r C_{\ell}^{\mathrm{T}}+C_{\ell}^{\mathrm{L}}}\right)^{2}\right]^{-1}
$$

Note that, to see the impact of the non-Gaussian property of the B-modes on the estimation of $\widehat{r}$ clearly, we ignore the instrumental noise in the observed B-modes to be used for estimating $r$. The effect of the instrumental noise on the estimation of $\widehat{r}$ is discussed later. The estimated tensor-to-scalar ratio would be close to that obtained from the maximum likelihood method 2 .

The histograms of the estimated tensor-to-scalar ratio, which are obtained from 10000 realizations of the tensor plus lensing and tensor plus delensed B-modes, are shown in Fig. 5. The input value of the tensor-to-scalar ratio is 0.05 . In both cases, the mean of the estimated tensor-to-scalar ratio equals to the input value within Monte Carlo error. Also in both cases, we find that the standard deviation of the tensor-to-scalar ratio increases only within a few percent $(\sim 2 \%)$ compared with that in the case of a Gaussian B-mode. Unlike the constraints on the power spectrum amplitude, the simulation results show that the standard deviation obtained in the presence of the lensing-induced non-Gaussian B-modes $\left(\sigma_{\text {sim }}^{r}\right)$ is not significantly different from that in the case of a Gaussian B-mode $\left(\sigma_{\mathrm{g}}^{r}\right)$. This is because well-defined estimators of $r$ usually extracts information mainly from large scale modes which earn a most part of the signal-to-noise of the tensor B-modes, while the B-modes on smaller

\footnotetext{
${ }^{2}$ Note that the estimator $\widehat{r}$ is not precisely the same as that obtained from the maximum likelihood method if the tensor-to-scalar ratio is close to zero and the fractional variance of $\widehat{r}$ is large. In our analysis here, the estimated values of the tensor-to-scalar ratio are much larger than the standard deviation.
} 
scales, where non-Gaussian properties of the lensing/delensed B-modes are significant, are down-weighted in the estimators. Note that the value of $\sigma_{\mathrm{sim}}^{r}\left(\sigma_{\mathrm{g}}^{r}\right)$ is $0.00075(0.00074)$ in the case of the lensing B-modes, and 0.00088 (0.00086) in the case of the delensed B-modes, respectively. That is, $\sigma_{\text {sim }}^{r}$ obtained in the case of the delensed B-modes is decreased by $\sim 15 \%$ compared to that in the case of the lensing B-modes, while $\sigma_{\mathrm{g}}^{r}$ is decreased by $14 \%$ after delensing. This means that the delensing efficiency is almost unchanged whether or not the non-Gaussian structure of the B-mode polarization is taken into account.

Let us discuss the case if the instrumental noise is added in the lensing and delensed B-modes. If a white noise is added, the B-modes at high multipoles behave like a Gaussian field, and the results of the estimation would be more close to those in the case of a Gaussian field. On the other hand, if the large scale B-modes are filtered out, the ratio of the standard deviations $\sigma_{\mathrm{sim}}^{r} / \sigma_{\mathrm{g}}^{r}$ would be increased. In practical situations, the large scale B-modes are significantly contaminated by Galactic foreground emission or $1 / \mathrm{f}$ noises. In Fig. 6 , the ratio of the simulated standard deviation to that in the case of a Gaussian field $\left(\sigma_{\mathrm{sim}}^{r} / \sigma_{\mathrm{g}}^{r}\right)$ is shown as a function of the minimum multipole included in the estimation $\left(\ell_{\min }\right)$. As expected, the ratio increases for larger values of $\ell_{\min }$. Also, the figure compares the constraint on the tensor-to-scalar ratio in the case of the tensor plus lensing B-modes with that in the case of the tensor plus delensed B-modes. Note that, if $\ell_{\min }$ is increased to be larger than 100 , the discrepancy between the two cases grows further.

\section{Summary and Discussion}

We have explored the non-Gaussian structure of the delensed B-mode polarization. Assuming an experiment of moderate sensitivity where the quadratic delensing is an almost optimal method, we find that not only the diagonal elements of the power spectrum covariance matrix but also its off-diagonal elements are reduced due to the delensing operation. In particular, the correlation coefficients between the power spectra at different multipoles become smaller than those of the original lensing B-modes. As a result, the constraint on the amplitude of the delensed B-mode power spectrum is not significantly degraded by the non-Gaussianity, compared to that on the amplitude of the lensing B-mode power spectrum. We show that the PDF of the power spectrum amplitude is broadened due to the non-Gaussianity, which leads to the increase of the variance, while the skewness and kurtosis of the PDF are basically consistent with those in the case of a Gaussian field. These features are well captured by the analytic model based on the 4th order Edgeworth expansion. Also, we show that the non-Gaussianity degrades the constraint on the tensor-to-scalar ratio after delensing within a few percent.

In this paper, we assumed the noise level of $\Delta_{\mathrm{P}}=6 \mu \mathrm{K}$-arcmin and the beam size of $\theta=4$ arcmin. In fact, our analytic model (and mathematical interpretation based on the model) works well as long as such moderate sensitivity experiments are assumed. For example, we checked that difference of $\sigma^{A}$ between simulations and the model prediction is $\sim 1 \%$ if $\Delta_{\mathrm{P}}=9 \mu \mathrm{K}$-arcmin or $7.5 \mu \mathrm{K}$-arcmin, and $\sim 2 \%$ if $\Delta_{\mathrm{P}}=3.5 \mu \mathrm{K}$-arcmin. On the other hand, decreasing the noise level, we found that the analytic calculations based on the formulas described in Sec. 4 eventually began to deviate from the simulation results. The ratio $\sigma^{A} / \sigma_{\mathrm{g}}^{A}$ increases after initial decrease in the simulation, while that of the analytic model decreases monotonically. For example, if the noise level is $1.5 \mu \mathrm{K}$-arcmin, the analytic model overestimates the constraint on the power spectrum amplitude by $\sim 7 \%$. In absence of instrumental noise (i.e., cosmic-variance limited up to $\ell=2000$ ), the discrepancy reaches to 
$\sim 14 \%$. This result implies that the approximations used in Sec. 4 are no longer valid. In such high sensitivity experiments, since quadratic delensing is no longer an optimal method, iterative delensing proposed in Ref. [42] would be used for actual delensing analysis. Properties of the B-modes delensed in such analysis should be explored through another simulation (and another analytic modeling) based on the iterative method. It would be our future work.

\section{Acknowledgments}

TN is supported by JSPS fellowship for abroad (Grant No. 26-142). We acknowledge the use of Healpix [47], Lenspix [37] and CAMB [36].

\section{A Statistical Properties of Power Spectrum Amplitude}

\section{A.1 Mean}

Let us first derive the mean of the power spectrum amplitude $\widehat{A}$. The mean of the power spectrum amplitude is given by

$$
\langle\widehat{A}\rangle=\langle\widehat{A}\rangle_{\mathrm{g}}+\langle\widehat{A} k(\boldsymbol{a})\rangle_{\mathrm{g}} .
$$

Since $\left\langle\left|a_{\ell m}\right|^{2}\right\rangle_{\mathrm{g}}=C_{\ell}$, the first term becomes

$$
\langle\widehat{A}\rangle_{\mathrm{g}}=\frac{\sum_{\ell m}\left(\left\langle\left|a_{\ell m}\right|^{2}\right\rangle_{\mathrm{g}} / C_{\ell}\right)}{\sum_{\ell}(2 \ell+1)}=1
$$

On the other hand, the second term vanishes. This is because, as described in Eq. (4.3), $k(\boldsymbol{a})$ is a linear combination of four derivatives with respect to $a_{\ell m}$. Therefore, for any $n$th order polynomial $f\left(a_{\ell m}\right)$ with $n<4$, a quantity $\left\langle f\left(a_{\ell m}\right) k(\boldsymbol{a})\right\rangle_{\mathrm{g}}$ vanishes by integration by parts. The mean of $\widehat{A}$ then becomes $\langle\widehat{A}\rangle=1$.

\section{A.2 Variance}

Next we derive the expression for the variance (4.9), and also Eq. (4.10). The variance of the amplitude estimator is defined as

$$
\left(\sigma^{A}\right)^{2} \equiv\left\langle\widehat{A}^{2}\right\rangle-1=\frac{1}{\left[\sum_{\ell}(2 \ell+1)\right]^{2}} \sum_{\ell \ell^{\prime}}(2 \ell+1)\left(2 \ell^{\prime}+1\right)\left(\left\langle\widehat{A}_{\ell} \widehat{A}_{\ell^{\prime}}\right\rangle-1\right) .
$$

Using the variance in the case of a Gaussian field $\left(\sigma_{\mathrm{g}}^{A}\right)^{2}$ defined in Eq. (3.7), the above equation becomes Eq. (4.9):

$$
\begin{aligned}
\left(\sigma^{A}\right)^{2} & =\frac{\left(\sigma_{\mathrm{g}}^{A}\right)^{2}}{n_{L}} \sum_{\ell \ell^{\prime}} \frac{(2 \ell+1)\left(2 \ell^{\prime}+1\right)}{2} \frac{\left\langle C_{\ell} C_{\ell^{\prime}}\right\rangle-C_{\ell} C_{\ell^{\prime}}}{C_{\ell} C_{\ell^{\prime}}} \\
& =\frac{\left(\sigma_{\mathrm{g}}^{A}\right)^{2}}{n_{L}} \sum_{\ell \ell^{\prime}} \frac{(2 \ell+1)\left(2 \ell^{\prime}+1\right)}{2} \frac{\operatorname{Cov}_{\ell \ell^{\prime}}}{C_{\ell} C_{\ell^{\prime}}} .
\end{aligned}
$$

Here, we denote the power spectrum covariance as

$$
\operatorname{Cov}_{\ell \ell^{\prime}}=\left\langle C_{\ell} C_{\ell^{\prime}}\right\rangle-C_{\ell} C_{\ell^{\prime}} .
$$


The power spectrum covariance is rewritten as

$$
\operatorname{Cov}_{\ell \ell^{\prime}}=\left\langle C_{\ell} C_{\ell^{\prime}}\right\rangle_{\mathrm{g}}-C_{\ell} C_{\ell^{\prime}}+\left\langle C_{\ell} C_{\ell^{\prime}} k(\boldsymbol{a})\right\rangle_{\mathrm{g}}
$$

The sum of the first two terms is the disconnected part of the power spectrum covariance:

$$
\operatorname{Cov}_{\ell \ell^{\prime}}^{\mathrm{g}} \equiv\left\langle C_{\ell} C_{\ell^{\prime}}\right\rangle_{\mathrm{g}}-C_{\ell} C_{\ell^{\prime}}=\frac{2 C_{\ell} C_{\ell^{\prime}}}{2 \ell+1} \delta_{\ell \ell^{\prime}}
$$

On the other hand, the connected part of the covariance is related to the trispectrum as (see equations from Eq. (A.10) to Eq. (A.14) for the derivation of Eq. (A.9) from Eq. (A.8))

$$
\begin{aligned}
\operatorname{Cov}_{\ell \ell^{\prime}}^{\mathrm{c}} & \equiv\left\langle C_{\ell} C_{\ell^{\prime}} k(\boldsymbol{a})\right\rangle_{\mathrm{g}} \\
& =\frac{1}{(2 \ell+1)\left(2 \ell^{\prime}+1\right)} \sum_{m m^{\prime}}\left\langle a_{\ell m} a_{\ell m}^{*} a_{\ell^{\prime} m^{\prime}} a_{\ell^{\prime} m^{\prime}}^{*} k(\boldsymbol{a})\right\rangle_{\mathrm{g}} \\
& =\frac{1}{(2 \ell+1)\left(2 \ell^{\prime}+1\right)} \sum_{m m^{\prime}}(-1)^{m+m^{\prime}} T_{m,-m, m^{\prime},-m^{\prime}}^{\ell \ell \ell^{\prime} \ell^{\prime}}
\end{aligned}
$$

The sum of the disconnected part (A.7) and connected part (A.9) gives Eq. (4.10).

The remaining part of this subsection is devoted for derivation of Eq. (A.9) from Eq. (A.8). Using Eq. (4.3), we rewrite Eq. (A.8) as

$$
\begin{aligned}
\frac{1}{(2 \ell+1)\left(2 \ell^{\prime}+1\right)} \sum_{m m^{\prime}} & \left\langle a_{\ell m} a_{\ell m}^{*} a_{\ell^{\prime} m^{\prime}} a_{\ell^{\prime} m^{\prime}}^{*} k(\boldsymbol{a})\right\rangle_{\mathrm{g}} \\
& =\frac{1}{(2 \ell+1)\left(2 \ell^{\prime}+1\right)} \sum_{m m^{\prime}} \int \mathrm{d} \boldsymbol{a} a_{\ell m} a_{\ell m}^{*} a_{\ell^{\prime} m^{\prime}} a_{\ell^{\prime} m^{\prime}}^{*} k(\boldsymbol{a}) P_{\mathrm{g}}(\boldsymbol{a}) \\
& =\frac{1}{(2 \ell+1)\left(2 \ell^{\prime}+1\right)} \sum_{m m^{\prime}} \frac{1}{24} \sum_{\ell_{i} m_{i}} T_{m_{1} m_{2} m_{3} m_{4}}^{\ell_{1} \ell_{2} \ell_{3} \ell_{4}} K
\end{aligned}
$$

where we define

$$
K \equiv \int \mathrm{d} \boldsymbol{a} a_{\ell m} a_{\ell m}^{*} a_{\ell^{\prime} m^{\prime}} a_{\ell^{\prime} m^{\prime}}^{*} \frac{\partial}{\partial a_{\ell_{1} m_{1}}} \frac{\partial}{\partial a_{\ell_{2} m_{2}}} \frac{\partial}{\partial a_{\ell_{3} m_{3}}} \frac{\partial}{\partial a_{\ell_{4} m_{4}}} P_{\mathrm{g}}(\boldsymbol{a}) .
$$

Introducing an operator $c_{i}$ which only affects on $a_{\ell_{i} m_{i}}$ as $c_{i} a_{\ell_{i} m_{i}}=a_{\ell_{i} m_{i}}^{*}$, we perform integration by parts which gives

$$
\begin{aligned}
K=- & \int \mathrm{d} \boldsymbol{a}\left[\delta_{\ell \ell_{1}} \delta_{m m_{1}}\left(a_{\ell m}^{*}+c_{1} a_{\ell m}\right) a_{\ell^{\prime} m^{\prime}} a_{\ell^{\prime} m^{\prime}}^{*}+\left(\ell m \leftrightarrow \ell^{\prime} m^{\prime}\right)\right] \\
& \times \frac{\partial}{\partial a_{\ell_{2} m_{2}}} \frac{\partial}{\partial a_{\ell_{3} m_{3}}} \frac{\partial}{\partial a_{\ell_{4} m_{4}}} P_{\mathrm{g}}(\boldsymbol{a})
\end{aligned}
$$


Repeating integration by parts, we find

$$
\begin{aligned}
K=\int & \mathrm{d} \boldsymbol{a}\left[\delta_{\ell \ell_{1}} \delta_{m m_{1}} \delta_{\ell \ell_{2}} \delta_{m m_{2}}\left(c_{2}+c_{1}\right) a_{\ell^{\prime} m^{\prime}} a_{\ell^{\prime} m^{\prime}}^{*}\right. \\
& \left.+\delta_{\ell \ell_{1}} \delta_{m m_{1}}\left(a_{\ell m}^{*}+c_{1} a_{\ell m}\right) \delta_{\ell^{\prime} \ell_{2}} \delta_{m^{\prime} m_{2}}\left(a_{\ell^{\prime} m^{\prime}}^{*}+c_{2} a_{\ell^{\prime} m^{\prime}}\right)+\left(\ell m \leftrightarrow \ell^{\prime} m^{\prime}\right)\right] \\
& \times \frac{\partial}{\partial a_{\ell_{3} m_{3}}} \frac{\partial}{\partial a_{\ell_{4} m_{4}}} P_{\mathrm{g}}(\boldsymbol{a}) \\
= & \int \mathrm{d} \boldsymbol{a}\left[\delta_{\ell \ell_{1}} \delta_{m m_{1}} \delta_{\ell \ell_{2}} \delta_{m m_{2}}\left(c_{2}+c_{1}\right) \delta_{\ell^{\prime} \ell_{3}} \delta_{m^{\prime} m_{3}}\left(a_{\ell^{\prime} m^{\prime}}^{*}+c_{3} a_{\ell^{\prime} m^{\prime}}\right)\right. \\
& +\delta_{\ell \ell_{1}} \delta_{m m_{1}} \delta_{\ell \ell_{3}} \delta_{m m_{3}}\left(c_{3}+c_{1}\right) \delta_{\ell^{\prime} \ell_{2}} \delta_{m^{\prime} m_{2}}\left(a_{\ell^{\prime} m^{\prime}}^{*}+c_{2} a_{\ell^{\prime} m^{\prime}}\right) \\
& \left.\left.+\delta_{\ell \ell_{1}} \delta_{m m_{1}}\left(a_{\ell m}^{*}+c_{1} a_{\ell m}\right) \delta_{\ell^{\prime} \ell_{2}} \delta_{m^{\prime} m_{2}} \delta_{\ell^{\prime} \ell_{3}} \delta_{m^{\prime} m_{3}}\left(c_{3}+c_{2}\right)\right]+\left(\ell m \leftrightarrow \ell^{\prime} m^{\prime}\right)\right] \\
& \times \frac{\partial}{\partial a_{\ell_{4} m_{4}}} P_{\mathrm{g}}(\boldsymbol{a}) \\
= & \mathrm{d} \boldsymbol{a} \frac{1}{4} \sum_{h, i, j, k=(1,2,3,4)} \delta_{\ell \ell_{h}} \delta_{m m_{h}} \delta_{\ell \ell_{i}} \delta_{m m_{i}} \delta_{\ell^{\prime} \ell_{j}} \delta_{m^{\prime} m_{j}} \delta_{\ell^{\prime} \ell_{k}} \delta_{m^{\prime} m_{k}}\left(c_{h}+c_{i}\right)\left(c_{j}+c_{k}\right) P_{\mathrm{g}}(\boldsymbol{a}) \\
= & \frac{1}{4} \sum_{h, i, j, k=(1,2,3,4)} \delta_{\ell \ell_{h}} \delta_{m m_{h}} \delta_{\ell \ell_{i}} \delta_{m m_{i}} \delta_{\ell^{\prime} \ell_{j}} \delta_{m^{\prime} m_{j}} \delta_{\ell^{\prime} \ell_{k}} \delta_{m^{\prime} m_{k}}\left(c_{h}+c_{i}\right)\left(c_{j}+c_{k}\right) .
\end{aligned}
$$

The summation with respect to $h, i, j$ and $k$ is applied for every permutaion of $(1,2,3,4)$. Using the above equation, we obtain

$$
\begin{aligned}
& \frac{1}{(2 \ell+1)\left(2 \ell^{\prime}+1\right)} \sum_{m m^{\prime}}\left\langle a_{\ell m} a_{\ell m}^{*} a_{\ell^{\prime} m^{\prime}} a_{\ell^{\prime} m^{\prime}}^{*} k(\boldsymbol{a})\right\rangle_{\mathrm{g}} \\
& =\frac{1}{(2 \ell+1)\left(2 \ell^{\prime}+1\right)} \sum_{m m^{\prime}}(-1)^{m+m^{\prime}} T_{m,-m, m^{\prime},-m^{\prime}}^{\ell \ell \ell^{\prime} \ell^{\prime}}
\end{aligned}
$$

This equation equals to Eq. (A.9).

\section{A.3 Probability distribution function of power spectrum amplitude}

Here we derive the PDF of the power spectrum amplitude, $\widehat{A}$, shown in Eq. (4.11). The PDF of $\widehat{A}$ is defined as

$$
\begin{aligned}
P(\widehat{A}) & \equiv \int \mathrm{d}^{n_{L}} \boldsymbol{a} \delta_{\mathrm{D}}\left(\widehat{A}-\frac{\sum_{\ell m}\left|a_{\ell m}\right|^{2} / C_{\ell}}{n_{L}}\right) P(\boldsymbol{a}) \\
& \propto \int \mathrm{d}^{n_{L}} \boldsymbol{a} \delta_{\mathrm{D}}\left(\widehat{A}-\frac{\sum_{\ell m}\left|a_{\ell m}\right|^{2} / C_{\ell}}{n_{L}}\right)[1+k(\boldsymbol{a})] \prod_{\ell^{\prime}} C_{\ell^{\prime}}^{-\frac{2 \ell^{\prime}+1}{2}} \exp \left[-\sum_{m^{\prime}=-\ell^{\prime}}^{\ell^{\prime}} \frac{\left|a_{\ell^{\prime} m^{\prime}}\right|^{2}}{2 C_{\ell^{\prime}}}\right] \\
& =\left[\prod_{\ell^{\prime}} C_{\ell^{\prime}}^{-\frac{2 \ell^{\prime}+1}{2}}\right] \mathrm{e}^{-\frac{n_{L}}{2} \widehat{A}} \int \mathrm{d}^{n_{L}} \boldsymbol{a} \delta_{\mathrm{D}}\left(\widehat{A}-\frac{\sum_{\ell m}\left|a_{\ell m}\right|^{2} / C_{\ell}}{n_{L}}\right)[1+k(\boldsymbol{a})] .
\end{aligned}
$$

Here $\delta_{\mathrm{D}}$ is the Dirac delta function. Introducing variables $u_{\ell m}$ so that $a_{\ell m}=\left(n_{L} \widehat{A} C_{\ell}\right)^{1 / 2} u_{\ell m}$, the above relation is simplified as

$$
\begin{aligned}
P(\widehat{A}) & =P_{\mathrm{g}}(\widehat{A}) \int \mathrm{d}^{n_{L}} \boldsymbol{u} \delta_{\mathrm{D}}\left(1-|\boldsymbol{u}|^{2}\right)[1+k(\boldsymbol{u}, \widehat{A})] \\
& \equiv P_{\mathrm{g}}(\widehat{A}) \mathcal{K}(\widehat{A})
\end{aligned}
$$


where the PDF in the case of a Gaussian field has the dependence as

$$
P_{\mathrm{g}}(\widehat{A}) \propto \widehat{A}^{\frac{n_{L}}{2}-1} \mathrm{e}^{-\frac{n_{L}}{2} \widehat{A}} .
$$

We define the correction factor as follows:

$$
\mathcal{K}(\widehat{A}) \equiv \frac{P(\widehat{A})}{P_{\mathrm{g}}(\widehat{A})}=\int \mathrm{d}^{n_{L}} \boldsymbol{u} \delta_{\mathrm{D}}\left(1-|\boldsymbol{u}|^{2}\right)+\int \mathrm{d}^{n_{L}} \boldsymbol{u} \delta_{\mathrm{D}}\left(1-|\boldsymbol{u}|^{2}\right) k(\boldsymbol{u}, \widehat{A}) .
$$

Let us consider a simplified expression for $\mathcal{K}(\widehat{A})$. For this purpose, we use the following formula

$$
\begin{aligned}
\int \mathrm{d}^{n_{L}} \boldsymbol{x} \delta_{\mathrm{D}}\left(1-|\boldsymbol{x}|^{2}\right) f\left(|\boldsymbol{x}|^{2}\right) & =S_{n_{L}} \int \mathrm{d} r r^{n_{L}-1} \delta_{\mathrm{D}}\left(1-r^{2}\right) f\left(r^{2}\right) \\
& =\frac{S_{n_{L}}}{2} \int \mathrm{d} s s^{\left(n_{L}-2\right) / 2} \delta_{\mathrm{D}}(1-s) f(s)=\frac{S_{n_{L}} f(1)}{2},
\end{aligned}
$$

where $S_{n_{L}}=2 \pi^{n_{L} / 2} / \Gamma\left(n_{L} / 2\right)$ is the surface area of the unit sphere in $n_{L}-1$ dimension. From the above equation, the first term of Eq. (A.18) is given by $S_{n_{L}} / 2$. On the other hand, the evaluation of the second term requires a bit complicated calculation. Using the explicit expression for the kurtosis contribution (4.4), we first rewrite the second term of Eq. (A.18) as

$$
\begin{gathered}
\frac{1}{24} \sum_{\ell_{i} m_{i}} \frac{T_{m_{1} m_{2} m_{3} m_{4}}^{\ell_{1} \ell_{2} \ell_{3} \ell_{4}}}{\left(C_{\ell_{1}} C_{\ell_{2}} C_{\ell_{3}} C_{\ell_{4}}\right)^{1 / 2}} \int \mathrm{d}^{n_{L}} \boldsymbol{u} \delta_{\mathrm{D}}\left(1-|\boldsymbol{u}|^{2}\right)\left(n_{L}^{2} \widehat{A}^{2} u_{\ell_{1} m_{1}}^{*} u_{\ell_{2} m_{2}}^{*} u_{\ell_{3} m_{3}}^{*} u_{\ell_{4} m_{4}}^{*}\right. \\
-6 n_{L} \widehat{A} \delta_{\ell_{1} \ell_{2}} \delta_{-m_{1}, m_{2}}(-1)^{m_{1}} u_{\ell_{3} m_{3}}^{*} u_{\ell_{4} m_{4}}^{*} \\
\left.+3 \delta_{\ell_{1} \ell_{2}} \delta_{-m_{1}, m_{2}} \delta_{\ell_{3} \ell_{4}} \delta_{-m_{3}, m_{4}}(-1)^{m_{1}+m_{3}}\right)
\end{gathered}
$$

Note that this is a real number for a given set of $a_{\ell m} \mathrm{~s}$. The above equation consists of the three terms: the term proportional to $\widehat{A}^{2}, \widehat{A}$ and independent of $\widehat{A}$. We first consider the term containing $\widehat{A}^{2}$. To simplify this term, we use the following general formula:

$$
\begin{aligned}
& \sum_{i j k m} \mathcal{T}_{i j k m} \int \mathrm{d}^{n_{L}} \boldsymbol{x} \delta_{\mathrm{D}}\left(1-|\boldsymbol{x}|^{2}\right) x_{i} x_{j} x_{k} x_{m} \\
& =\int \mathrm{d}^{n_{L}} \boldsymbol{x} \delta_{\mathrm{D}}\left(1-|\boldsymbol{x}|^{2}\right)\left(\sum_{i} \mathcal{T}_{i i i i}\left|x_{i}\right|^{4}+3 \sum_{i \neq j} \mathcal{T}_{i j i j}\left|x_{i}\right|^{2}\left|x_{j}\right|^{2}\right) \\
& =3 I_{4} \sum_{i j} \mathcal{T}_{i j i j} .
\end{aligned}
$$

Here, the quantity $\mathcal{T}_{i j k l}$ is assumed to be unchanged by the exchange of two idices, e.g. $\mathcal{T}_{i j k l}=\mathcal{T}_{j i k l}$. We define $I_{4}$ as

$$
I_{4} \equiv \int \mathrm{d}^{n_{L}} \boldsymbol{x} \delta_{\mathrm{D}}\left(1-|\boldsymbol{x}|^{2}\right)\left|x_{i}\right|^{2}\left|x_{j}\right|^{2},
$$

where $i \neq j$. Also, we use the fact that (see Sec. A.4 for derivation):

$$
I_{4}=\frac{1}{3} \int \mathrm{d}^{n_{L}} \boldsymbol{x} \delta_{\mathrm{D}}\left(1-|\boldsymbol{x}|^{2}\right)\left|x_{i}\right|^{4} .
$$


$I_{4}$ is independent of the indices ( $i$ and $j$ ) appearing in the r.h.s. of the above equations. The quantity $I_{4}$ is computed as

$$
\begin{aligned}
\left(3 \sum_{i}+\sum_{i \neq j}\right) I_{4} & =\int \mathrm{d}^{n_{L}} \boldsymbol{x} \delta_{\mathrm{D}}\left(1-|\boldsymbol{x}|^{2}\right)\left(\sum_{i}\left|x_{i}\right|^{4}+\sum_{i \neq j}\left|x_{i}\right|^{2}\left|x_{j}\right|^{2}\right) \\
& =\int \mathrm{d}^{n_{L}} \boldsymbol{x} \delta_{\mathrm{D}}\left(1-|\boldsymbol{x}|^{2}\right)\left(\sum_{i}\left|x_{i}\right|^{2}\right)^{2}=\frac{S_{n_{L}}}{2} .
\end{aligned}
$$

By use of Eqs. (A.21) and (A.24), the first term of Eq. (A.20) is given by

$$
\begin{aligned}
& \frac{1}{24} \sum_{\ell_{i} m_{i}} \frac{T_{m_{1} m_{2} m_{3} m_{4}}^{\ell_{1} \ell_{2} \ell_{3} \ell_{4}}}{\left(C_{\ell_{1}} C_{\ell_{2}} C_{\ell_{3}} C_{\ell_{4}}\right)^{1 / 2}} \int \mathrm{d}^{n_{L}} \boldsymbol{u} \delta_{\mathrm{D}}\left(1-|\boldsymbol{u}|^{2}\right) n_{L}^{2} \widehat{A}^{2} u_{\ell_{1} m_{1}}^{*} u_{\ell_{2} m_{2}}^{*} u_{\ell_{3} m_{3}}^{*} u_{\ell_{4} m_{4}}^{*} \\
& =S_{n_{L}} \frac{n_{L}}{n_{L}+2} \frac{\widehat{A}^{2}}{16} \sum_{\ell m \ell^{\prime} m^{\prime}} \frac{\left\langle\left|a_{\ell m}\right|^{2}\left|a_{\ell^{\prime} m^{\prime}}\right|^{2}\right\rangle_{c}}{C_{\ell} C_{\ell^{\prime}}} .
\end{aligned}
$$

Next we consider the second term of Eq. (A.20). Similarly as in the case of the first term, we define the following integral:

$$
I_{2} \equiv \int \mathrm{d}^{n_{L}} \boldsymbol{u} \delta_{\mathrm{D}}\left(1-|\boldsymbol{u}|^{2}\right) u_{i} u_{j}=\delta_{i j} \int \mathrm{d}^{n_{L}} \boldsymbol{u} \delta_{\mathrm{D}}\left(1-|\boldsymbol{u}|^{2}\right)\left|u_{i}\right|^{2} .
$$

This quantity satisfies

$$
\sum_{i} I_{2}=\int \mathrm{d}^{n_{L}} \boldsymbol{x} \delta_{\mathrm{D}}\left(1-|\boldsymbol{x}|^{2}\right) \sum_{i}\left|x_{i}\right|^{2}=\frac{S_{n_{L}}}{2} .
$$

This reduces the second term of Eq. (A.20) to

$$
\begin{aligned}
& \frac{-\widehat{A}}{4} \sum_{\ell_{i} m_{i}} T_{m_{1} m_{2} m_{3} m_{4}}^{\ell_{1} \ell_{2} \ell_{3} \ell_{4}} \int \mathrm{d}^{n_{L}} \boldsymbol{u} \delta_{\mathrm{D}}\left(1-|\boldsymbol{u}|^{2}\right) \delta_{\ell_{1} \ell_{2}} \delta_{-m_{1}, m_{2}}(-1)^{m_{1}} u_{\ell_{3} m_{3}}^{*} u_{\ell_{4} m_{4}}^{*} \\
& =-S_{n_{L}} \frac{\widehat{A}}{8} \sum_{\ell m \ell^{\prime} m^{\prime}} \frac{\left\langle\left|a_{\ell m}\right|^{2}\left|a_{\ell^{\prime} m^{\prime}}\right|^{2}\right\rangle_{c}}{C_{\ell} C_{\ell^{\prime}}} .
\end{aligned}
$$

Finally, the last term becomes

$$
\begin{aligned}
& \frac{1}{8} \sum_{\ell_{i} m_{i}} T_{m_{1} m_{2} m_{3} m_{4}}^{\ell_{1} \ell_{2} \ell_{3} \ell_{4}} \int \mathrm{d}^{n_{L}} \boldsymbol{u} \delta_{\mathrm{D}}\left(1-|\boldsymbol{u}|^{2}\right) \delta_{\ell_{1} \ell_{2}} \delta_{-m_{1}, m_{2}} \delta_{\ell_{3} \ell_{4}} \delta_{-m_{3}, m_{4}}(-1)^{m_{1}+m_{3}} \\
& =\frac{S_{n_{L}}}{16} \sum_{\ell m \ell^{\prime} m^{\prime}} \frac{\left\langle\left|a_{\ell m}\right|^{2}\left|a_{\ell^{\prime} m^{\prime}}\right|^{2}\right\rangle_{c}}{C_{\ell} C_{\ell^{\prime}}} .
\end{aligned}
$$

Combining these results, we find

$$
\mathcal{K}(\widehat{A})=\frac{S_{n_{L}}}{2}+S_{n_{L}} \frac{\left(n_{L} /\left(n_{L}+2\right) \widehat{A}^{2}-2 \widehat{A}+1\right)}{16} \sum_{\ell m \ell^{\prime} m^{\prime}} \frac{\left\langle\left|a_{\ell m}\right|^{2}\left|a_{\ell^{\prime} m^{\prime}}\right|^{2}\right\rangle_{c}}{C_{\ell} C_{\ell^{\prime}}} .
$$

We finally obtain the PDF of $\widehat{A}$ as

$$
P(\widehat{A}) \propto \widehat{A}^{\frac{n_{L}}{2}-1} \mathrm{e}^{-\frac{n_{L}}{2} \widehat{A}}\left(1+\frac{\left(n_{L}\right) /\left(n_{L}+2\right) \widehat{A}^{2}-2 \widehat{A}+1}{8} \sum_{\ell \ell^{\prime}}(2 \ell+1)\left(2 \ell^{\prime}+1\right) \frac{\operatorname{Cov}_{\ell \ell^{\prime}}^{\mathrm{c}}}{C_{\ell} C_{\ell^{\prime}}}\right) .
$$

Note that the normalization is given by $\left(n_{L} / 2\right)^{n_{L} / 2} / \Gamma\left(n_{L} / 2\right)$. 


\section{A.4 Formula of integration on unit hypersurface}

Finally, we derive the relationship between the following two integrals:

$$
\begin{aligned}
J_{i j} & \equiv \int \mathrm{d}^{n} \boldsymbol{x} \delta_{\mathrm{D}}\left(1-|\boldsymbol{x}|^{2}\right)\left|x_{i}\right|^{2}\left|x_{j}\right|^{2}, \\
J_{i} & \equiv \int \mathrm{d}^{n} \boldsymbol{x} \delta_{\mathrm{D}}\left(1-|\boldsymbol{x}|^{2}\right)\left|x_{i}\right|^{4} .
\end{aligned}
$$

In our derivation, we use

$$
F(\boldsymbol{t}) \equiv \int \mathrm{d}^{n} \boldsymbol{x} \delta_{\mathrm{D}}\left(1-|\boldsymbol{x}|^{2}\right) \mathrm{e}^{-\left(t_{1} x_{1}^{2}+t_{2} x_{2}^{2}+\cdots+t_{n} x_{n}^{2}\right)},
$$

where $\boldsymbol{t}^{t}=\left(t_{1}, t_{2}, \cdots, t_{n}\right)$ are non-negative parameters. This function is considered as a generating function of $J_{i j}$ and $J_{i}$, since these quantities are given as a derivative of $F(\boldsymbol{t})$ in terms of $t_{i}$ :

$$
J_{i j}=\lim _{\boldsymbol{t} \rightarrow+0} \frac{\partial^{2} F(\boldsymbol{t})}{\partial t_{i} \partial t_{j}}, \quad J_{i}=\lim _{\boldsymbol{t} \rightarrow+0} \frac{\partial^{2} F(\boldsymbol{t})}{\partial t_{i}^{2}} .
$$

The generating function (A.34) is rewritten as

$$
\begin{aligned}
F(\boldsymbol{t}) & =\int \mathrm{d}^{n} \boldsymbol{x} \int \mathrm{d} s \frac{1}{2 \pi} \mathrm{e}^{-\mathrm{i} s\left(1-|\boldsymbol{x}|^{2}\right)} \mathrm{e}^{-\left(t_{1} x_{1}^{2}+t_{2} x_{2}^{2}+\cdots+t_{n} x_{n}^{2}\right)} \\
& =\int \mathrm{d} s \frac{1}{2 \pi} \mathrm{e}^{-\mathrm{i} s} \prod_{i=1}^{n} \int \mathrm{d} x_{i} \exp \left[-\left(t_{i}-\mathrm{i} s\right) x_{i}^{2}\right] \\
& =\int \mathrm{d} s \frac{1}{2 \pi} \mathrm{e}^{-\mathrm{i} s} \prod_{i=1}^{n}\left(\frac{\pi}{t_{i}-\mathrm{i} s}\right)^{1 / 2} .
\end{aligned}
$$

Substituting the above equation into Eq. (A.35), we find

$$
3 J_{i j}=J_{i} .
$$

Since $I_{4}=J_{i j}$, we obtain Eq. (A.24).

\section{B Delensed B-mode Power Spectrum Covariance}

\section{B.1 Analytic expression}

Let us discuss analytic expression for the power spectrum covariance of the delensed B-mode polarization. The estimator of the delensed B-mode polarization is given by Eq. (2.19). For a realization of our simulation, the lensing potential is obtained from the simulated CMB map according to Eqs. (2.11) and (2.13). The estimated lensing potential is decomposed into the lensing potential and the other remaining term (the noise of the lensing reconstruction) as

$$
\widehat{\phi}_{\ell m}=\phi_{\ell m}+n_{\ell m} .
$$

From Eq. (2.16), the estimator of the lensing B-mode is described as

$$
\mathscr{B}_{\ell m}\left[E, \phi^{\mathrm{w}}\right]+\mathscr{B}_{\ell m}\left[E, n^{\mathrm{w}}\right],
$$


where the Wiener filtered multipoles $\phi_{\ell m}^{\mathrm{w}}$ and $n_{\ell m}^{\mathrm{w}}$ are defined by multiplying the Wiener filter $W_{\ell}^{\phi}$ in Eq. (2.18) to the $\phi_{\ell m}$ and $n_{\ell m}$, respectively. The dominant contribution of the lensing B-mode at large scales is also expressed by the convolution of the E-mode polarization and lensing potential as described in Eq. (2.6) [10]. On the assumption that the lensing Bmode is given by Eq. (2.6), the delensed B-mode polarization (in the absence of the tensor perturbations) is described as a sum of two components:

$$
B_{\ell m}^{\mathrm{d}}=\mathscr{B}_{\ell m}\left[E,\left(1-W^{\phi}\right) \phi\right]-\mathscr{B}_{\ell m}\left[E, n^{\mathrm{w}}\right] .
$$

In the above equations, we assume that the Wiener filter for the E-mode $\left(W^{\mathrm{E}}\right)$ is unity up to $\ell=2000$, i.e., uncertainty in the E-mode polarization is dominated by its cosmic variance and the instrumental noise is negligible. This approximation would be valid for ongoing and future high-resolution experiments since their polarization sensitivity will be better than $\mathcal{O}(1) \mu \mathrm{K}$-arcmin also with small beam sizes of a few arcminute. The filter function for the lensing potential $\left(W_{\ell}^{\phi}\right)$ depends on the lensing power spectrum of the theoretical model which is identified through a parameter estimation procedure in actual analysis. We assume that the model is correctly identified in our simulation and the filter function is henceforth treated as a quantity independent of $C_{\ell}^{\phi \phi}$.

The delensed B-mode power spectrum is computed from Eq. (B.3). Under the assumption that the lensing potential $\phi_{\ell m}$ is statistically independent from the residual reconstruction noise $n_{\ell m}$, the delensed B-mode power spectrum becomes [10]

$$
\begin{aligned}
C_{\ell}^{\mathrm{BB}, \mathrm{d}} & \simeq \Xi_{\ell}\left[C^{\mathrm{EE}},\left(1-W^{\phi}\right)^{2} C^{\phi \phi}\right]+\Xi_{\ell}\left[C^{\mathrm{EE}},\left(W^{\phi}\right)^{2} N^{\phi \phi}\right] \\
& \simeq \Xi_{\ell}\left[C^{\mathrm{EE}},\left(1-W^{\phi}\right) C^{\phi \phi}\right],
\end{aligned}
$$

where $N_{\ell}^{\phi \phi}$ is the power spectrum of $n_{\ell m}$, and, from Eq. (B.4) to (B.5), we assume that $N_{\ell}^{\phi \phi}$ corresponds to $A_{\ell}^{\phi}$ given in Eq. (2.14). Note that, strictly speaking, the lensing potential and reconstruction noise are correlated because the reconstruction noise has contributions from the lensing potential and lensed E-mode. The impact of the correlation between $\phi_{\ell m}$ and $n_{\ell m}$ would be, however, negligible at least for experiments assumed in this paper, since the approximate form of Eq. (B.5) is in good agreement with the simulation results. This issue is also discussed in our previous work [34] where we assumed the similar experimental specification.

To derive the power spectrum covariance in the delensed case, we need to compute the correlation of Eq. (B.4). Denoting the first and second terms of Eq. (B.4) as

$$
\begin{aligned}
D_{\ell}^{\phi} & \equiv \Xi_{\ell}\left[C^{\mathrm{EE}},\left(1-W^{\phi}\right)^{2} C^{\phi \phi}\right], \\
D_{\ell}^{n} & \equiv \Xi_{\ell}\left[C^{\mathrm{EE}},\left(W^{\phi}\right)^{2} N^{\phi \phi}\right],
\end{aligned}
$$

we write the covariance of the delensed B-mode power spectrum as the sum of the following three terms:

$$
\begin{aligned}
T_{\ell \ell^{\prime}}^{4 \phi} & =\left\langle D_{\ell}^{\phi} D_{\ell^{\prime}}^{\phi}\right\rangle, \\
T_{\ell \ell^{\prime}}^{4 n} & =\left\langle D_{\ell}^{n} D_{\ell^{\prime}}^{n}\right\rangle, \\
T_{\ell \ell^{\prime}}^{2 \phi 2 n} & =\left\langle D_{\ell}^{\phi} D_{\ell^{\prime}}^{n}\right\rangle+\left(\ell \leftrightarrow \ell^{\prime}\right) .
\end{aligned}
$$


The first two terms would be evaluated analytically with the analogy used in the case of the lensing B-modes [31, 32], i.e.,

$$
\begin{aligned}
T_{\ell \ell^{\prime}}^{4 \phi} & =\sum_{L} \frac{2}{2 L+1}\left\{\frac{\partial D_{\ell}^{\phi}}{\partial \ln C_{L}^{\mathrm{EE}}} \frac{\partial D_{\ell^{\prime}}^{\phi}}{\partial \ln C_{L}^{\mathrm{EE}}}+\frac{\partial D_{\ell}^{\phi}}{\partial \ln C_{L}^{\phi \phi}} \frac{\partial D_{\ell^{\prime}}^{\phi}}{\partial \ln C_{L}^{\phi \phi}}\right\} \\
& =\sum_{L} \frac{2}{2 L+1}\left\{\frac{\partial D_{\ell}^{\phi}}{\partial \ln C_{L}^{\mathrm{EE}}} \frac{\partial D_{\ell^{\prime}}^{\phi}}{\partial \ln C_{L}^{\mathrm{EE}}}+\left(1-W_{L}^{\phi}\right)^{4} \frac{\partial \widetilde{C}_{\ell}^{\mathrm{BB}}}{\partial \ln C_{L}^{\phi \phi}} \frac{\partial \widetilde{C}_{\ell^{\prime}}^{\mathrm{BB}}}{\partial \ln C_{L}^{\phi \phi}}\right\},
\end{aligned}
$$

and

$$
T_{\ell \ell^{\prime}}^{4 n}=\sum_{L} \frac{2}{2 L+1}\left\{\frac{\partial D_{\ell}^{n}}{\partial \ln C_{L}^{\mathrm{EE}}} \frac{\partial D_{\ell^{\prime}}^{n}}{\partial \ln C_{L}^{\mathrm{EE}}}+\left[\frac{\left(W_{L}^{\phi}\right)^{2} N_{L}^{\phi \phi}}{C_{L}^{\phi \phi}}\right]^{2} \frac{\partial \widetilde{C}_{\ell}^{\mathrm{BB}}}{\partial \ln C_{L}^{\phi \phi}} \frac{\partial \widetilde{C}_{\ell^{\prime}}^{\mathrm{BB}}}{\partial \ln C_{L}^{\phi \phi}}\right\}
$$

Here we assume that the power spectra $C^{\mathrm{EE}}, C^{\phi \phi}$ and $N^{\phi \phi}$ have Gaussian covariance. Note that, as discussed in Refs. [20, 48, 49], the covariance between $C^{\mathrm{EE}}, C^{\phi \phi}$ and $N^{\phi \phi}$ can have off-diagonal elements and is shown that these are basically negligible. The remaining term $T_{\ell \ell^{\prime}}^{2 \phi 2 n}$ would be approximated as

$$
\begin{aligned}
T_{\ell \ell^{\prime}}^{2 \phi 2 n}=\sum_{L} & \frac{2}{2 L+1}\left\{\frac{\partial D_{\ell}^{\phi}}{\partial \ln C_{L}^{\mathrm{EE}}} \frac{\partial D_{\ell^{\prime}}^{n}}{\partial \ln C_{L}^{\mathrm{EE}}}\right. \\
& \left.+\left(1-W_{L}^{\phi}\right)^{2}\left(W_{L}^{\phi}\right)^{2} \frac{N_{L}^{\phi \phi}}{C_{L}^{\phi \phi}} \frac{\partial \widetilde{C}_{\ell}^{\mathrm{BB}}}{\partial \ln C_{L}^{\phi \phi}} \frac{\partial \widetilde{C}_{\ell^{\prime}}^{\mathrm{BB}}}{\partial \ln C_{L}^{\phi \phi}}\right\}+\left(\ell \leftrightarrow \ell^{\prime}\right) .
\end{aligned}
$$

By combining the above three terms, we find

$$
\begin{aligned}
T_{\ell \ell^{\prime}}^{4 \phi}+T_{\ell \ell^{\prime}}^{4 n}+T_{\ell \ell^{\prime}}^{2 \phi 2 n}= & \sum_{L} \frac{2}{2 L+1}\left\{\frac{\partial\left(D_{\ell}^{\phi}+D_{\ell}^{n}\right)}{\partial \ln C_{L}^{\mathrm{EE}}} \frac{\partial\left(D_{\ell^{\prime}}^{\phi}+D_{\ell^{\prime}}^{n}\right)}{\partial \ln C_{L}^{\mathrm{EE}}}\right. \\
& \left.+\left[\left(1-W_{L}^{\phi}\right)^{2}+\frac{\left(W_{L}^{\phi}\right)^{2} N_{L}^{\phi \phi}}{C_{L}^{\phi \phi}}\right]^{2} \frac{\partial \widetilde{C}_{\ell}^{\mathrm{BB}}}{\partial \ln C_{L}^{\phi \phi}} \frac{\partial \widetilde{C}_{\ell^{\prime}}^{\mathrm{BB}}}{\partial \ln C_{L}^{\phi \phi}}\right\} \\
\simeq & \sum_{L} \frac{2}{2 L+1}\left\{\frac{\partial\left(D_{\ell}^{\phi}+D_{\ell}^{n}\right)}{\partial \ln C_{L}^{\mathrm{EE}}} \frac{\partial\left(D_{\ell^{\prime}}^{\phi}+D_{\ell^{\prime}}^{n}\right)}{\partial \ln C_{L}^{\mathrm{EE}}}\right. \\
& \left.+\left(1-W_{L}^{\phi}\right)^{2} \frac{\partial \widetilde{C}_{\ell}^{\mathrm{BB}}}{\partial \ln C_{L}^{\phi \phi}} \frac{\partial \widetilde{C}_{\ell^{\prime}}^{\mathrm{BB}}}{\partial \ln C_{L}^{\phi \phi}}\right\} \\
\simeq & \sum_{L} \frac{2}{2 L+1}\left\{\frac{\partial C_{\ell}^{\mathrm{BB}, \mathrm{d}}}{\partial \ln C_{L}^{\mathrm{EE}}} \frac{\partial C_{\ell^{\prime}}^{\mathrm{BB}, \mathrm{d}}}{\partial \ln C_{L}^{\mathrm{EE}}}+\frac{\partial C_{\ell}^{\mathrm{BB}, \mathrm{d}}}{\partial \ln C_{L}^{\phi \phi}} \frac{\partial C_{\ell^{\prime}}^{\mathrm{BB}, \mathrm{d}}}{\partial \ln C_{L}^{\phi \phi}}\right\} .
\end{aligned}
$$

From the first to second equation, we assume that $N_{\ell}^{\phi \phi}$ corresponds to $A^{\phi}$, and from the second to third equation, we use Eq. (B.5). The covariance of the delensed B-mode power spectrum given above equals to the off-diagonal part of Eq. (4.18). Note that we compute the derivative of the delensed B-mode power spectrum $C_{\ell}^{\mathrm{BB}, \mathrm{d}}$ with respect to $C_{\ell}^{\phi \phi}$ via Eq. (B.5). 


\section{B.2 Numerical computation}

Here we describe our method of computing the power spectrum covariance of the lensing and delensed B-mode. The power spectrum covariance is given by

$$
\operatorname{Cov}_{\ell \ell^{\prime}}^{\mathrm{BB}}=\frac{2}{2 \ell+1} C_{\ell}^{2} \delta_{\ell \ell^{\prime}}+\operatorname{Cov}_{\ell \ell^{\prime}}^{\mathrm{E}}+\operatorname{Cov}_{\ell \ell^{\prime}}^{\phi},
$$

where we denote the connected part of the covariance as

$$
\begin{aligned}
& \operatorname{Cov}_{\ell \ell^{\prime}}^{\mathrm{E}}=\sum_{L} \frac{\partial C_{\ell}}{\partial C_{L}^{\mathrm{EE}}} \frac{2\left(C_{L}^{\mathrm{EE}}\right)^{2}}{2 L+1} \frac{\partial C_{\ell^{\prime}}}{\partial C_{L}^{\mathrm{EE}}}, \\
& \operatorname{Cov}_{\ell \ell^{\prime}}^{\phi}=\sum_{L} \frac{\partial C_{\ell}}{\partial C_{L}^{\phi \phi}} \frac{2\left(C_{L}^{\phi \phi}\right)^{2}}{2 L+1} \frac{\partial C_{\ell^{\prime}}}{\partial C_{L}^{\phi \phi}} .
\end{aligned}
$$

To evaluate the connected part of the covariance, $\mathrm{Cov}^{\mathrm{E}}$ and $\mathrm{Cov}^{\phi}$, we rewrite the derivatives as

$$
\begin{gathered}
\frac{\partial C_{\ell}}{\partial C_{L}^{\mathrm{EE}}}=\Xi_{\ell L}^{\phi}\left[C^{\phi \phi}\right], \\
\frac{\partial C_{\ell}}{\partial C_{L}^{\phi \phi}}=\Xi_{\ell L}^{\mathrm{E}}\left[C^{\mathrm{EE}}\right] .
\end{gathered}
$$

Here we define

$$
\begin{aligned}
& \Xi_{\ell L}^{\phi}[A] \equiv \frac{1}{2 \ell+1} \sum_{L^{\prime}}\left(\mathcal{S}_{\ell L L^{\prime}}^{(-)}\right)^{2} A_{L^{\prime}}, \\
& \Xi_{\ell L}^{\mathrm{E}}[A] \equiv \frac{1}{2 \ell+1} \sum_{L^{\prime}}\left(\mathcal{S}_{\ell L^{\prime} L}^{(-)}\right)^{2} A_{L^{\prime}} .
\end{aligned}
$$

Note that

$$
\Xi_{\ell}[A, B]=\sum_{L} A_{L} \Xi_{\ell L}^{\phi}[B]=\sum_{L} B_{L} \Xi_{\ell L}^{\mathrm{E}}[A]
$$

For instance, $\operatorname{Cov}_{\ell \ell^{\prime}}^{\mathrm{E}}$ is then given by

$$
\begin{aligned}
\operatorname{Cov}_{\ell \ell^{\prime}}^{\mathrm{E}} & =\sum_{L} \frac{\partial C_{\ell}}{\partial C_{L}^{\mathrm{EE}}} \frac{2\left(C_{L}^{\mathrm{EE}}\right)^{2}}{2 L+1} \frac{\partial C_{\ell^{\prime}}}{\partial C_{L}^{\mathrm{EE}}} \\
& =\sum_{L} \Xi_{\ell L}^{\phi}\left[C^{\phi \phi}\right] \frac{2\left(C_{L}^{\mathrm{EE}}\right)^{2}}{2 L+1} \Xi_{\ell^{\prime} L}^{\phi}\left[C^{\phi \phi}\right] .
\end{aligned}
$$

Denoting

$$
F_{L}^{\ell^{\prime}}=\frac{2\left(C_{L}^{\mathrm{EE}}\right)^{2}}{2 L+1} \Xi_{\ell^{\prime} L}^{\phi}\left[C^{\phi \phi}\right]
$$

we obtain

$$
\operatorname{Cov}_{\ell \ell^{\prime}}^{\mathrm{E}}=\Xi_{\ell}\left[F^{\ell^{\prime}}, C^{\phi \phi}\right] .
$$


Similarly, we find

$$
\operatorname{Cov}_{\ell \ell^{\prime}}^{\phi}=\Xi_{\ell}\left[C^{\mathrm{EE}}, G^{\ell^{\prime}}\right]
$$

where we define

$$
G_{L}^{\ell^{\prime}}=\frac{2\left(C_{L}^{\phi \phi}\right)^{2}}{2 L+1} \Xi_{\ell^{\prime} L}^{\mathrm{E}}\left[C^{\mathrm{EE}}\right]
$$

The summations in $\Xi_{\ell}, \Xi_{\ell L}^{\mathrm{E}}$ and $\Xi_{\ell L}^{\phi}$ are efficiently evaluated by use of the reduced wigner $\mathrm{d}$ functions as described in Ref. [10]. The power spectrum covariance of the delensed B-modes is also evaluated in the above similar manner.

\section{References}

[1] BICEP2 and PLANCK Collaboration, "A Joint Analysis of BICEP2/Keck Array and Planck Data", Phys. Rev. Lett. (2015) [arXiv:1502.00612].

[2] Planck Collaboration, "Planck 2015 results. XIII. Cosmological parameters", arXiv: 1502.01589.

[3] J. Dunkley et al., "CMBPol Mission Concept Study: Prospects for polarized foreground removal", AIP Conf. Proc. 1141 (2009) 222, [arXiv:0811.3915].

[4] M. Betoule, E. Pierpaoli, J. Delabrouille, M. Le Jeune, and J.-F. Cardoso, "Measuring the tensor to scalar ratio from CMB B-modes in the presence of foregrounds", Astronomy and Astrophysics 503 (sep, 2009) 691-706, [arXiv:0901.1056].

[5] K. Ichiki, "CMB foreground: A concise review", Prog. Theor. Exp. Phys. 06 (2014) B109.

[6] N. Katayama and E. Komatsu, "Simple foreground cleaning algorithm for detecting primordial B-mode polarization of the cosmic microwave background", Astrophys. J. 737 (2011) 78, [arXiv: 1101.5210].

[7] M. Zaldarriaga and U. Seljak, "Gravitational lensing effect on cosmic microwave background polarization", Phys. Rev. D 58 (1998) 023003, [astro-ph/9803150].

[8] A. Lewis and A. Challinor, "Weak gravitational lensing of the CMB", Phys. Rept. 429 (2006) 1-65, [astro-ph/0601594].

[9] U. Seljak and C. M. Hirata, "Gravitational lensing as a contaminant of the gravity wave signal in CMB", Phys. Rev. D 69 (2004) 043005, [astro-ph/0310163].

[10] K. M. Smith et al., "Delensing CMB Polarization with External Datasets", JCAP 1206 (2012) 014, [arXiv: 1010.0048].

[11] L. Boyle, K. M. Smith, C. Dvorkin, and N. Turok, "On testing and extending the inflationary consistency relation for tensor modes", arXiv: 1408.3129.

[12] G. Simard, D. Hanson, and G. Holder, "Prospects for Delensing the Cosmic Microwave Background for Studying Inflation", arXiv:1410.0691.

[13] T. Namikawa, D. Yamauchi, and A. Taruya, "Future detectability of gravitational-wave induced lensing from high-sensitivity CMB experiments", Phys. Rev. D 91 (2015), no. 4 043531, [arXiv:1411.7427].

[14] W.-H. Teng, C.-L. Kuo, and J.-H. P. Wu, "Cosmic Microwave Background Delensing Revisited: Residual Biases and a Simple Fix", arXiv:1102.5729.

[15] K. Kamada, Y. Miyamoto, D. Yamauchi, and J. Yokoyama, "Effects of cosmic strings with delayed scaling on CMB anisotropy", Phys. Rev. D 90 (2014), no. 8083502 , [arXiv: 1407.2951]. 
[16] I. D. Saltas, I. Sawicki, L. Amendola, and M. Kunz, "Anisotropic stress as signature of non-standard propagation of gravitational waves", arXiv:1406.7139.

[17] E. Fenu, D. G. Figueroa, R. Durrer, and J. Garcia-Bellido, "Gravitational waves from self-ordering scalar fields", JCAP 10 (oct, 2009) 5, [arXiv:0908.0425].

[18] D. G. Figueroa, M. Hindmarsh, and J. Urrestilla, "Exact Scale-Invariant Background of Gravitational Waves from Cosmic Defects", Physical Review Letters 110 (mar, 2013) 101302, [arXiv: 1212.5458].

[19] ACT Collaboration, A. van Engelen et al., "The Atacama Cosmology Telescope: Lensing of CMB Temperature and Polarization Derived from Cosmic Infrared Background Cross-Correlation", arXiv:1412.0626.

[20] Planck Collaboration , "Planck 2015 results. XV. Gravitational lensing", arXiv:1502.01591.

[21] POLARBEAR Collaboration, "Measurement of the Cosmic Microwave Background Polarization Lensing Power Spectrum with the POLARBEAR experiment", Phys.Rev.Lett. 113 (2014) 021301, [arXiv:1312.6646].

[22] SPT Collaboration, D. Hanson et al., "Detection of B-mode Polarization in the Cosmic Microwave Background with Data from the South Pole Telescope", Phys. Rev. Lett. 111 (2013) 141301, [arXiv:1307.5830].

[23] SPT Collaboration, K. T. Story et al., "A Measurement of the Cosmic Microwave Background Gravitational Lensing Potential from 100 Square Degrees of SPTpol Data", arXiv:1412.4760.

[24] E. Calabrese et al., "Precision Epoch of Reionization studies with next-generation CMB experiments", JCAP 1408 (2014) 010, [arXiv:1406.4794].

[25] K. Arnold et al., "The Simons Array: expanding POLARBEAR to three multi-chroic telescopes", Proc. SPIE 91531 (2014) 91531F.

[26] B. A. Benson, "SPT-3G: A Next-Generation Cosmic Microwave Background Polarization Experiment on the South Pole Telescope", Proceedings of SPIE (2014) [arXiv:1407.2973].

[27] K. Abazajian et al., "Inflation Physics from the Cosmic Microwave Background and Large Scale Structure", Astropart. Phys. 63 (2015) 55-65, [arXiv:1309.5381].

[28] B. D. Sherwin and M. Schmittfull, "Delensing the CMB with the Cosmic Infrared Background", arXiv: 1502.05356.

[29] K. M. Smith, W. Hu, and M. Kaplinghat, "Weak lensing of the CMB: Sampling errors on B-modes", Phys. Rev. D 70 (2004) 043002, [astro-ph/0402442].

[30] K. M. Smith, W. Hu, and M. Kaplinghat, "Cosmological Information from Lensed CMB Power Spectra", Phys. Rev. D 74 (2006) 123002, [astro-ph/0607315].

[31] C. Li, T. L. Smith, and A. Cooray, "Non-Gaussian Covariance of CMB B-modes of Polarization and Parameter Degradation", Phys. Rev. D 75 (2007) 083501, [astro-ph/0607494].

[32] A. Benoit-Lévy, K. M. Smith, and W. Hu, "Non-Gaussian structure of the lensed CMB power spectra covariance matrix", Phys. Rev. D 86 (2012), no. 12 123008, [arXiv:1205.0474].

[33] Planck Collaboration, "Planck 2013 results. XVI. Cosmological parameters", Astron. Astrophys. 571 (2014) A16, [arXiv:1303.5076].

[34] T. Namikawa and R. Nagata, "Lensing reconstruction from a patchwork of polarization maps", JCAP 1409 (2014) 009, [arXiv: 1405.6568].

[35] T. Namikawa, D. Yamauchi, and A. Taruya, "Full-sky lensing reconstruction of gradient and 
curl modes from CMB maps", JCAP 1201 (2012) 007, [arXiv:1110.1718].

[36] A. Lewis, A. Challinor, and A. Lasenby, "Efficient Computation of CMB anisotropies in closed FRW models", Astrophys. J. 538 (2000) 473-476, [astro-ph/9911177].

[37] A. Challinor and A. Lewis, "Lensed CMB power spectra from all-sky correlation functions", Phys. Rev. D 71 (2005) 103010, [astro-ph/0502425].

[38] W. Hu, "Weak lensing of the CMB: A harmonic approach", Phys. Rev. D 62 (2000) 043007, [astro-ph/0001303].

[39] T. Okamoto and W. Hu, "CMB Lensing Reconstruction on the Full Sky", Phys. Rev. D 67 (2003) 083002, [astro-ph/0301031].

[40] D. Hanson et al., "CMB temperature lensing power reconstruction", Phys. Rev. D $\mathbf{8 3}$ (2011) 043005, [arXiv: 1008.4403].

[41] A. Lewis, A. Challinor, and D. Hanson, "The shape of the CMB lensing bispectrum", JCAP 1103 (2011) 018, [arXiv:1101.2234].

[42] C. M. Hirata and U. Seljak, "Reconstruction of lensing from the cosmic microwave background polarization", Phys. Rev. D 68 (2003) 083002, [astro-ph/0306354].

[43] S. Hamimeche and A. Lewis, "Likelihood Analysis of CMB Temperature and Polarization Power Spectra", Phys. Rev. D 77 (2008) 103013, [arXiv:0801.0554].

[44] D. M. Regan, E. P. S. Shellard, and J. R. Fergusson, "General CMB and primordial trispectrum estimation", Phys. Rev. D 82 (jul, 2010) 023520, [arXiv:1004.2915].

[45] T. Namikawa, D. Hanson, and R. Takahashi, "Bias-Hardened CMB Lensing", Mon. Not. Roy. Astron. Soc. 431 (2013) 609-620, [arXiv:1209.0091].

[46] T. Namikawa and R. Takahashi, "Bias-Hardened CMB Lensing with Polarization", Mon. Not. Roy. Astron. Soc. 438 (2014), no. 2 1507-1517, [arXiv:1310.2372].

[47] K. Gorski et al., "HEALPix - A Framework for high resolution discretization, and fast analysis of data distributed on the sphere", Astrophys. J. 622 (2005) 759-771, [astro-ph/0409513].

[48] M. M. Schmittfull, A. Challinor, D. Hanson, and A. Lewis, "Joint analysis of CMB temperature and lensing-reconstruction power spectra", Phys. Rev. D $8 \mathbf{8}$ (2013), no. 6063012 , [arXiv: 1308.0286].

[49] Planck Collaboration, "Planck 2013 results. XVII. Gravitational lensing by large-scale structure", Astron. Astrophys. 571 (2014) A17, [arXiv:1303.5077]. 